THE AstrophysicAl JOURNAL, IN PRESS

Preprint typeset using LTEX style emulateapj v. 08/22/09

\title{
SUPERNOVA 1996cr: SN 1987A’S WILD COUSIN?
}

\author{
F. E. BAuer, ${ }^{1}$ V. V. Dwarkadas,${ }^{2}$ W. N. Brandt ${ }^{3}$ S. Immler,${ }^{4}$ S. SMartt,${ }^{5}$ N. Bartel,${ }^{6}$ And M. F. BIEtenholz ${ }^{6,7}$ \\ (Received 2007 November 14; Accepted 2008 April 21) \\ The Astrophysical Journal, in press
}

\begin{abstract}
We report on new VLT optical spectroscopic and multi-wavelength archival observations of SN 1996cr, a previously identified ultraluminous X-ray source known as Circinus Galaxy X-2. The spectrum of the optical counterpart confirms SN 1996cr as a bona fide type IIn SN, in accordance with its tentative SN classification at X-ray wavelengths. SN 1996cr is one of the closest $\mathrm{SNe}(\approx 3.8 \mathrm{Mpc})$ in the last several decades and in terms of flux ranks among the brightest radio and X-ray SNe ever detected. Optical imaging from the Anglo-Australian Telescope archive allows us to isolate the explosion date to between 1995-02-28 and 1996-03-16, while the wealth of optical, X-ray, and radio observations that exist for this source provide relatively detailed constraints on its post-explosion expansion and progenitor history, including an preliminary angular size constraint from VLBI. Archival X-ray and radio data imply that the progenitor of SN 1996cr evacuated a large cavity just prior to exploding via either a sped-up wind or a pre-SN explosion. The blast wave likely spent $\sim 1-2$ yrs in relatively uninhibited expansion before eventually striking the dense circumstellar material which surrounds SN 1996cr to become a prodigious X-ray and radio emitter. The X-ray and radio emission, which trace the progenitor mass-loss rate, have respectively risen by a factor of $\gtrsim 2$ and remained roughly constant over the past $\approx 7$ yr. This behavior is reminiscent of the late rise of SN 1987A, but three orders of magnitude more luminous and much more rapid to onset. SN 1996cr may likewise provide us with a younger example of SN 1978K and SN 1979C, both of which exhibit flat X-ray evolution at late times. The optical spectrum suggests that the progenitor was a massive star that shed some of its outer envelope (many $\mathbf{M}_{\odot}$ ) prior to explosion, while the complex Oxygen line emission hints at a possible concentric shell or ring-like structure. Taken together, this implies that a substantial fraction of the closest SNe observed in the last several decades have occurred in wind-blown bubbles, and argues for the phenomena being widespread.
\end{abstract}

Subject headings: stars: supernovae: general — stars: circumstellar matter — X-rays: supernovae — radio: supernovae -

\section{INTRODUCTION}

Although thousands of supernovae ( $\mathrm{SNe}$ ) have been discovered to date, only several dozen have been detected at either X-ray ${ }^{8}$ or radio 9 wavelengths (designated XSNe or RSNe, respectively). Many of these XSNe and RSNe were originally detected simply as variable X-ray or radio sources, and only verified as true SNe after careful examination of archival optical data or through optical follow-up (e.g., Rupen et al. 1987; Ryder et al. 1993). This particular path to discovery has meant that only a handful of these sources have been well-studied during the first several hundred days, a period which is critical for identifying and characterizing the true nature of the SNe. ${ }^{10}$

\footnotetext{
${ }^{1}$ Chandra Fellow, Columbia Astrophysics Laboratory, 550 W. 120th St., Columbia University, New York, NY 10027; feb@astro.columbia.edu

${ }^{2}$ Department of Astronomy and Astrophysics, University of Chicago, 5640 South Ellis Avenue, RI 451, Chicago, IL 60637

${ }^{3}$ Department of Astronomy \& Astrophysics, The Pennsylvania State University, 525 Davey Lab, University Park, PA 16802.

${ }^{4}$ Goddard Space Flight Center, Code 662, Greenbelt, MD 20771, USA

${ }^{5}$ Astrophysics Research Centre, School of Maths and Physics, Queen's University Belfast, Belfast, BT7 1NN Northern Ireland, UK

${ }^{6}$ Department of Physics and Astronomy, York University, Toronto, ON M3J 1P3, Canada

${ }^{7}$ Hartebeesthoek Radio Observatory, PO Box 443, Krugersdorp, 1740, South Africa

${ }^{8}$ See http://lheawww.gsfc.nasa.gov/users/immler/supernovae_list.html for a complete list of X-ray SNe and references

${ }^{9}$ See http://rsd-www.nrl.navy.mil/7213/weiler/kwdata/RSNtable.txt for a list of radio $\mathrm{SNe}$ and references

${ }^{10}$ By contrast, XSNe and RSNe do comprise a substantial fraction of all $\mathrm{SNe}$ well-studied beyond a few hundred days, as such SNe end up being
}

Such SNe typically turn out to be core-collapse SNe of either type Ibc, which have been associated with long-duration gamma-ray bursts (GRBs), or type II, whereby the intense $\mathrm{X}$-ray and/or radio emission is thought to stem from the interaction between the expanding shock and a dense progenitor wind. When well-sampled, the additional multi-wavelength constraints from XSNe and RSNe can provide physical insights into the late evolutionary stages of massive stars that are otherwise impossible to obtain. For instance, while robust constraints on the overall time-averaged mass-loss in various phases of stellar evolution exist, there has been a longstanding debate over the number and sequence of various evolutionary stages for massive stars, and relatively few observational constraints on the actual evolution of mass-loss within many particular stages (e.g., Lamers et al. 1991; Langer et al. 1994; Stothers \& Chin 1996; Maeder \& Meynet 2000). Because the SN blast wave travels orders of magnitude faster than the stellar wind, studying the interaction between the blast wave and the progenitor stellar wind allows us to probe tens of thousands of years of evolution in a matter of decades. $\mathrm{SNe}$ progenitors are considered to provide the bulk of all processed stellar material additionally, and thus the characterization of these sources can likewise help further our understanding of overall galactic chemical enrichment.

Here we report on the spectroscopic confirmation of one such serendipitous source, SN 1996cr (Bauer 2007), and present a multi-wavelength follow-up study using archival data to determine its explosion date and temporal properties.

nearby and remain relatively bright for years to decades. 
SN 1996cr was originally detected as Circinus Galaxy (CG) $\mathrm{X}-2$, an ultraluminous $\mathrm{X}$-ray source in the nearby Circinus Galaxy (Freeman et al. 1977), which Bauer et al. (2001) found to exhibit many characteristics of a young, type II SN enshrouded in a dense circumstellar environment. In particular, it demonstrated a factor of $>30$ increase in X-ray flux between 1997-2000, a $k T \sim 10 \mathrm{keV}$ thermal spectrum with a strong, blended Fe emission-line component at $6.85 \mathrm{keV}$, and spatial coincidence with a strong radio and $\mathrm{H} \alpha$-emitting point source. The proximity of this SN affords us a rare opportunity to study in detail a type II SN which is strongly interacting with its circumstellar medium.

This paper is organized as follows: data and reduction methods are detailed in $\S 2$; confirmation of SN $1996 \mathrm{cr}$ as a SN and isolation of its explosion date are provided in $\S 3$; overall temporal and spectroscopic constraints for SN 1996cr are investigated in $\S 4$; and finally conclusions and future prospects are explored in $\$ 5$. Throughout this paper, we adopt a distance of $3.8 \pm 0.8 \mathrm{Mpc}$ to the Circinus Galaxy (converted from Freeman et al. 1977). While the Circinus Galaxy lies close to the Galactic Plane $(|b|=3.8)$, it is located within a Galactic "window" with a visual extinction of $A_{V}=1.5 \pm 0.2$ and a neutral hydrogen column density of $N_{\mathrm{H}}=(3.0 \pm 0.3) \times 10^{21}$ $\mathrm{cm}^{-2}$ (whereas neighboring regions typically have $A_{V}=3.0$ and $N_{\mathrm{H}}=(5-10) \times 10^{21} \mathrm{~cm}^{-2}$; Schlegel et al. 1998; Dickey \& Lockman 1990). Due to its $\sim 65^{\circ}$ inclination, however, there is significant internal obscuration as well $\left[N_{\mathrm{H}} \sim(5-8) \times 10^{21}\right.$ $\mathrm{cm}^{-2}$ typically; Bauer et al. 2001].

\section{OBSERVATIONAL DATA AND REDUCTION METHODS}

We describe below the extensive observational data used to constrain the properties of SN 1996cr. Fortunately, plentiful archival data exist for the Circinus Galaxy due to the fact that it hosts the second closest Compton-thick active galactic nuclei (AGN) to our own Galaxy and exhibits signs of vigorous star formation (e.g., Matt et al. 1999). The resulting multiwavelength dataset provides good constraints on the explosion date and yields useful long-term X-ray and radio light curves. SN 1996cr lies $25^{\prime \prime}$ to the south of the Circinus Galaxy nucleus. We adopt the position of $\alpha=14^{\mathrm{h}} 13^{m} 10^{\mathrm{s}} 01 \delta=-65^{\circ} 20^{\prime} 44^{\prime \prime} .4$ (J2000), determined from the radio observations.

\subsection{VLT Spectroscopy}

SN 1996cr was visited several times in service mode with the VLT FORS I spectrograph in two separate campaigns. The first program was initiated over the course of 2005-0306 through 2005-03-17 but never completed, resulting in a low signal-to-noise spectrum. As such, we do not provide any further details. The second program was executed over three nights (2006-01-26, 2006-02-02, and 2006-03-10), yielding a high-quality spectrum confirming the ambiguous features seen in the spectrum from our first program. Our analyses focus only on the spectrum from the first night, as the observations on the subsequent two nights suffer from significantly worse seeing ( $\approx 1 .{ }^{\prime \prime} 5-2 !^{\prime \prime} 0$ ) such that the co-addition of these frames failed to improve the signal-to-noise of the final spectrum. Our observations on the first night consisted of four $1200 \mathrm{~s}$ exposures taken with the $300 \mathrm{~V}$ grism and the TEK (24 $\mu$ pixel) CCD using a slitwidth of 2 !'$^{\prime \prime}$. The grism and CCD combination provided a dispersion of $2.66 \AA$ pixel $^{-1}$ and a total useful wavelength coverage of 3600-7990 $\AA$. These images were taken in excellent seeing conditions, in which stellar sources had a full-width half-maximum (FWHM) measured at $0 . " 6-0 . / 7$ on the FORS I chip over the course of the night. Our target, SN 1996cr, had an $\approx 20 \%$ larger FWHM image size than the point sources in the acquisition image, suggesting that it may be spatially extended or possibly contaminated by a coincident $\mathrm{H}$ II region. We investigated this further by measuring the FWHM orthogonal to the dispersion axis for the strong emission lines [O III] $\lambda 5007$ and $\mathrm{H} \alpha$, which had sizes of $0 .^{\prime \prime} 74$ and $0 .^{\prime \prime} 85$, respectively. The inherent FWHM slowly increased with decreasing wavelength, such that the FWHMs quoted above were $5 \pm 3 \%$ and $35 \pm 4 \%$ larger than their stellar equivalents, respectively. Thus the [O III] $\lambda 5007$ FWHM is formally unresolved, while the $\mathrm{H} \alpha$ line is extended. The strength, extent, and visible asymmetry in the FWHM profile of the extended $\mathrm{H} \alpha$ are fully consistent with the underlying $\mathrm{H}$ II region (see $\S 3$ for details), although we caution that marginally-resolved light echoes could potentially contaminate and broaden the PSF (e.g., Sugerman 2003). The spectral resolution $\left(11 \AA\right.$, or $\left.500 \mathrm{~km} \mathrm{~s}^{-1}\right)$ was es-

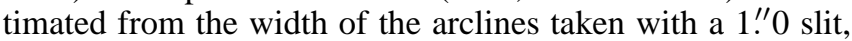
which matched closely the on-sky image size of SN 1996cr. After bias subtraction and flat-fielding using standard techniques, the co-added spectra were extracted, wavelength calibrated, and flux calibrated (using the standard LTT4816). Fig. 1 shows the resulting optical spectrum from 2006-01-26. We conservatively estimate the flux errors on the spectrum at $20 \%$.

\subsection{Archival Optical Data}

We searched through the European Southern Observatory (ESO), Anglo Australian Telescope (AAT), United Kingdom Schmidt Telescope (UKST), and Hubble Space Telescope (HST) archives. We only list here data that ultimately were used to constrain the explosion date of SN 1996cr. Relevant data are listed in Tables 1-4. Aperture photometry was performed throughout, with PSF-fitting employed as a crosscheck; there is only one instance where the PSF-fitting magnitude differed from the aperture-measured value at $\gtrsim 1 \sigma$, which we explicitly document below.

\subsection{1. $E S O$}

The Circinus Galaxy was observed extensively with SUSI at the NTT on 1993 April 9 using several narrow-band filters (\#369:[O III], \#430:5100 A continuum, \#629:H $\alpha+[\mathrm{N} \mathrm{II}]$,

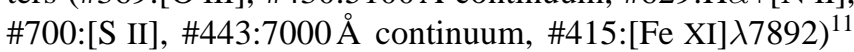
as well as with the IRAC2 camera at the ESO/MPI $2.2 \mathrm{~m}$ telescope on 1994 June 25 using broad-band $J$ and $H$ filters. Reduced images were kindly provided by E. Oliva and A. Marconi (private communication, 2007), for which cutouts of SN 1996cr are shown in Fig. 2. Details of the observations and reduction procedures are given in Marconi et al. (1994). The [O III], H $\alpha+[\mathrm{N} \mathrm{II}]$, and [S II] images were the only images provided to us in a flux-calibrated state, and therefore are the only ones for which we measure photometry. Magnitudes for the region in the vicinity of SN 1996cr were measured using a $1 . " 0$ radius circular aperture and previously established zero points. An aperture correction of 0.2 mags was estimated empirically using several bright, isolated point sources in the images. The resulting aperture-corrected magnitudes are presented in Table 1.

\footnotetext{
${ }^{11} \mathrm{http}: / /$ filters.ls.eso.org/efs/efs_fi.htm
} 


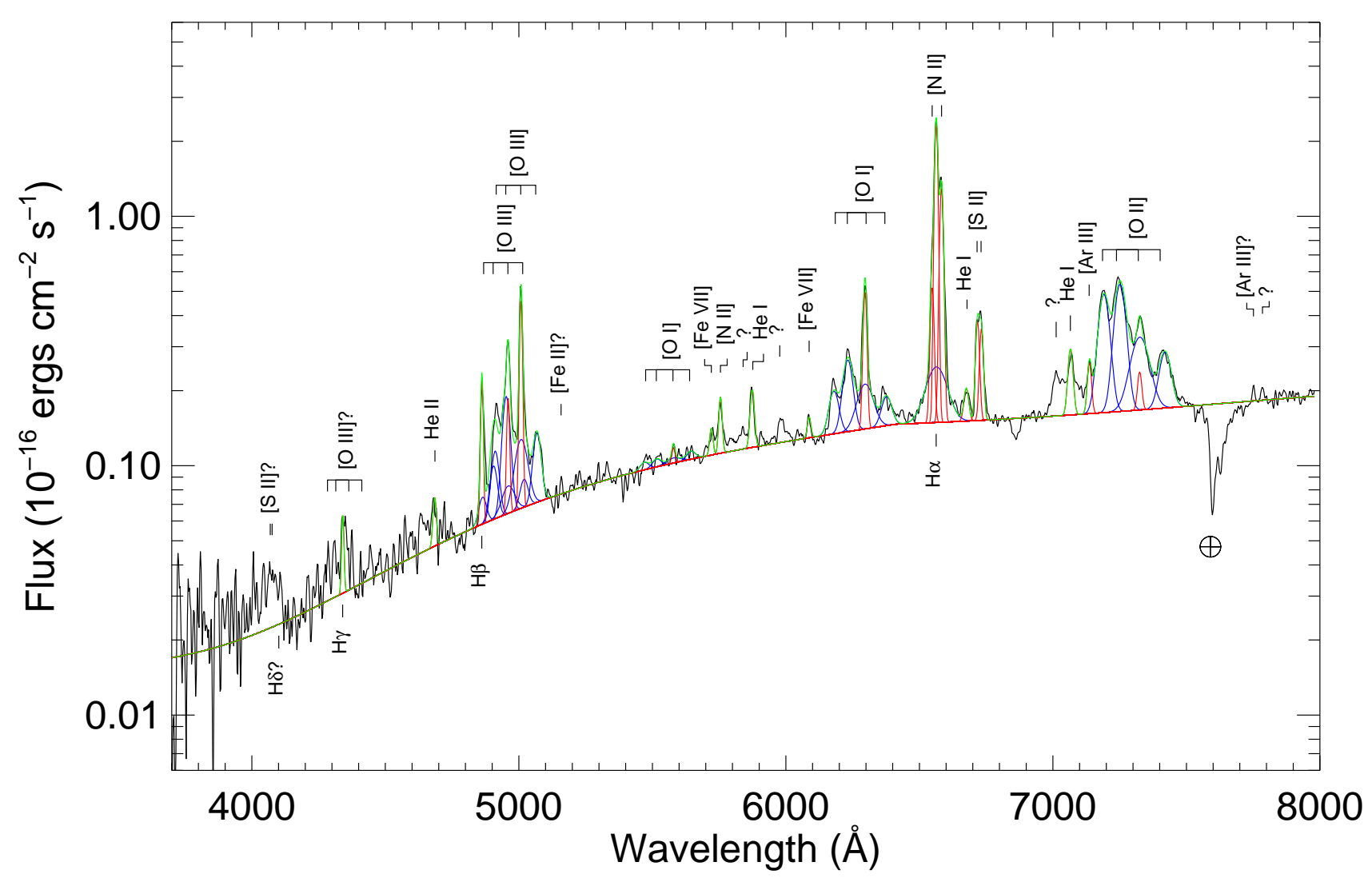

FIG. 1.- Flux-calibrated VLT FORS spectrum of SN 1996cr from 2006 January 26. The spectrum suffers from an extinction of $A_{V} \sim 4-6$, which we have not corrected. Obvious emission lines have been identified. There is strong, narrow $\mathrm{H} \alpha$ emission (FWHM $\approx 714 \mathrm{~km} \mathrm{~s}^{-1}$ ) as well as several broad $\mathrm{O}$ complexes showing a distinct underlying velocity structure. An empirical model has been fit to the data as described in $\S 4.2$; the green line shows the overall fit to the spectrum while the red (blue) lines show the individual narrow (broad) components. Strong, broad unidentified residuals remain around the He I lines ( $\lambda \lambda 5876,7065)$; this emission is likely associated with the structure of a He shell or the progenitor wind, but is not easily disentangled.

\subsubsection{HST}

The Circinus Galaxy was observed with both the WFPC2 and NICMOS instruments aboard HST on four separate occasions, as outlined in Table 2. Details of the photometric and astrometric reduction of the WFPC2 data are given in Bauer et al. (2001). Briefly, after standard calibration of the HST images, we used the IRAF package DAOPHOT to measure aperture-corrected magnitudes using a $0 .{ }^{\prime \prime} 2$ radius aperture for all sources down to the $2 \sigma$ detection limit. The HST images were then aligned to the Hipparcos/Tycho astrometric reference frame to $\approx 0 . \prime 4$. Due to the negligible overlap of the F606W observation with the other filters and complete lack of coverage of SN 1996cr itself, we do not discuss it further. For the NICMOS data, of which only the NIC3 images provided useful imaging, we used the standard pipeline data products. We performed photometry using a $0 .{ }^{\prime \prime} 5$ radius aperture and applied band-dependent aperture corrections determined from a set of isolated, unsaturated point sources in the vicinity of SN 1996cr. Cutout images of SN 1996cr are presented in Fig. 3. Notably, SN 1996cr appears to lie at the center of a diffuse patch of $\mathrm{H} \alpha$ emission in the F656N image and our aperture magnitude here differs from a PSF-fitting one at $\gtrsim 3 \sigma$ (0.11 magnitudes). We thus adopt the PSF-fitting magnitude, as well as an additional systematic error of 0.1 magnitudes to reflect the larger uncertainty associated with the deblending.

\subsection{3. $A A T$}

The Circinus Galaxy was observed numerous times with the TAURUS Fabry-Perot instrument on the AAT during 199502-21 to $1995-02-28$ and $1996-03-15$ to $1996-03-20$. The data were retrieved through the AAT archive. ${ }^{12}$ The observations were comprised primarily of narrow-band imaging and TAURUS spectral imaging cubes centered on the He II $\lambda 4686,[\mathrm{O}$ III] $\lambda \lambda 5007,4959, \mathrm{H} \alpha+[\mathrm{N}$ II] $\lambda \lambda 6583,6548$, and $[\mathrm{S}$ II] $\lambda \lambda \lambda 6731,6716$ emission lines. The original investigators discuss their observational design in Veilleux \& BlandHawthorn (1997) and Elmouttie et al. (1998), while more complete data reduction procedures are outlined in Gordon et al. (2000). To summarize, the TAURUS instrument was used in the angstrom imaging mode, wherein narrow-band filters were used at different tilt angles to isolate the lines of He II, [O III], H $\alpha+[\mathrm{N} \mathrm{II}]$, and [S II] (Bland-Hawthorn \& Jones 1998). Each square pixel subtended $0 .{ }^{\prime \prime} 315$ on the sky and the atmospheric seeing at FWHM averaged $\approx 1$ !' $2-2$ !' 0 . Unfortunately, a significant portion of the archived TAURUS images for the Circinus Galaxy have only limited accompanying CCD calibration data; there were often no obvious superbias frames, dark frames, dome-flats, or flux standards, so the photometric quality of the reduced data is limited.

Our best-effort reduction proceeded as follows. The overscan regions were used to subtract the bias from each frame

\footnotetext{
12 http://site.aao.gov.au/arc-bin/wdb/aat_database/observation_log/make
} 


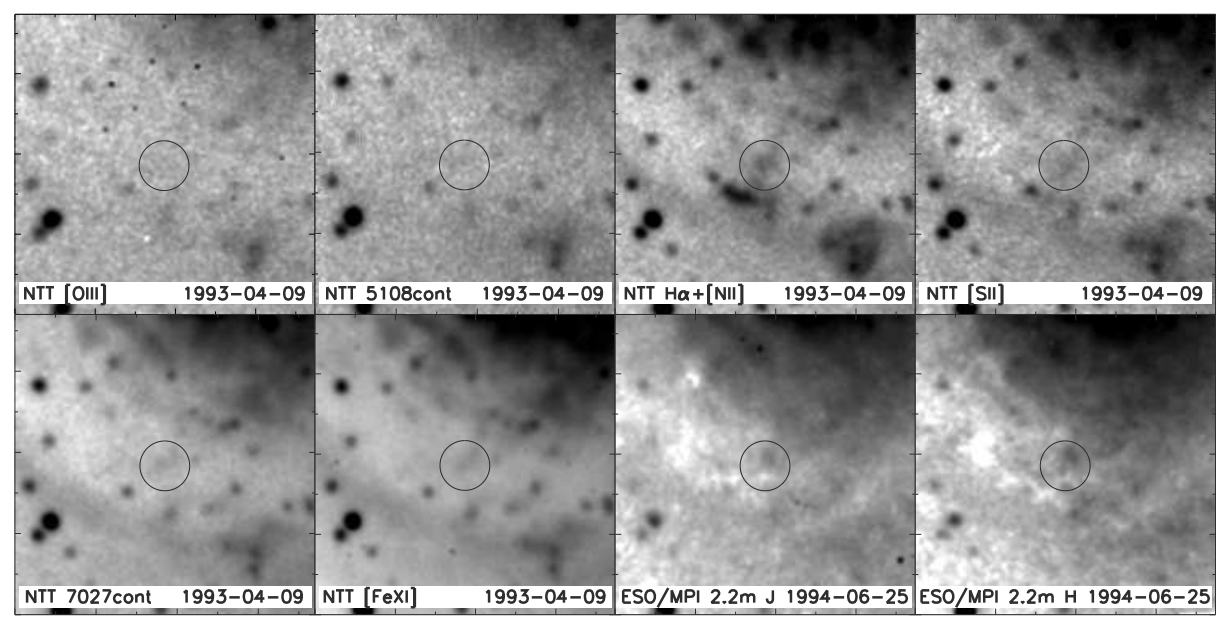

FIG. 2.- Postage-stamp images centered on SN 1996cr from ESO imaging observations in 1993-1994 courtesy of E. Oliva and A. Marconi (private communication, 2007). Images are $24^{\prime \prime}$ on a side. The position of SN $1996 \mathrm{cr}$ is denoted by a $2^{\prime \prime}$ radius circle. Each image is labeled, denoting both the instrument and filter used to acquire it and the observation date.

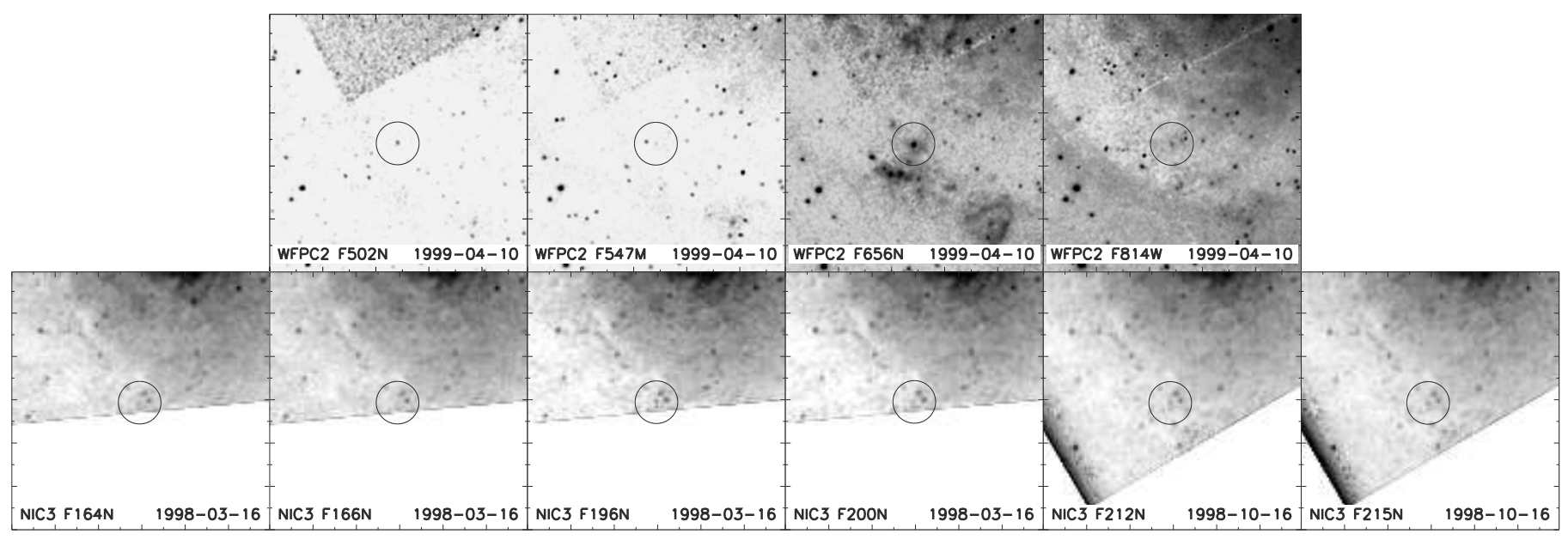

FIG. 3.- Postage-stamp images centered on SN 1996cr from HST WFPC2 and NIC3 imaging observations spanning 1996 to 1999 . SN $1996 \mathrm{cr}$ appears as a strong, unresolved emission-line source in the F502N and F656N images, but displays only weak continuum as evidenced its unremarkable appearance in all of the other HST images and the fainter broad-band magnitudes in Table 2. See Fig. 2 caption for details of image properties.

and each image was flat-fielded using a sky flat. When multiple frames in a given filter were available, the images were combined to reject cosmic rays using the IRAF task CRREJ. Source detection and photometry were performed with SExtractor (Bertin \& Arnouts 1996). A set of 40 relatively bright, isolated, unsaturated stars, which are detected in all the AAT and HST images, were chosen to register the AAT images to the HST coordinate frame. The alignment of each AAT im-

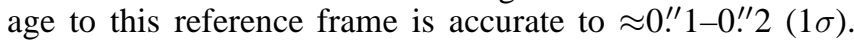
The zeropoints for the narrow-band images were obtained by bootstrapping the AAT magnitudes to the well-determined HST ones. To this end, HST colors were obtained for the 40 stars and matched to standard main sequence stellar templates from the Bruzual Atlas, ${ }^{13}$ modified by a standard Galactic dust model with $E(B-V)=1$ (the extinction measured toward the Circinus Galaxy; e.g., Schlegel et al. 1998) using the IRAF package SYNPHOT. Among the 40 calibration stars, 25 had colors consistent with one of the stellar templates.

\footnotetext{
${ }^{13}$ http://www.stsci.edu/hst/observatory/cdbs/bz77.html
}

These particular stellar templates and the TAURUS-specific transmission curves ${ }^{14}$ were input into SYNPHOT to convert between HST and TAURUS magnitudes. We used the IRAF task FITPARAMS to compare interactively the HST-derived magnitudes to the SExtractor ones and derive reasonable zeropoints for each image. We typically rejected a few outliers in FITPARAMS to obtain an adequate fit $(0.3-0.5 \mathrm{mag} 1 \sigma$ error typically on the zeropoint). The He II, [O III], and [S II] bands were observed multiple times, so we combined zeropoint estimates to ensure that magnitudes for the detected sources were consistent to within $\approx 0.1-0.2$ mags across these images. For bands which sampled comparable spectral windows, we used SYNPHOT to convert from one band to another (for instance, from TAURUS $5020 \AA / 30 \AA$ to HST F502N or from the VLT FORS spectrum to all overlapping bands). The resulting magnitudes are listed in Table 3, while Fig. 4 shows 12 images taken in 1995 February and 1996 March.

\footnotetext{
${ }^{14} \mathrm{http} / / /$ www.aao.gov.au/local/www/cgt/ccdimguide/filtercat.html
} 


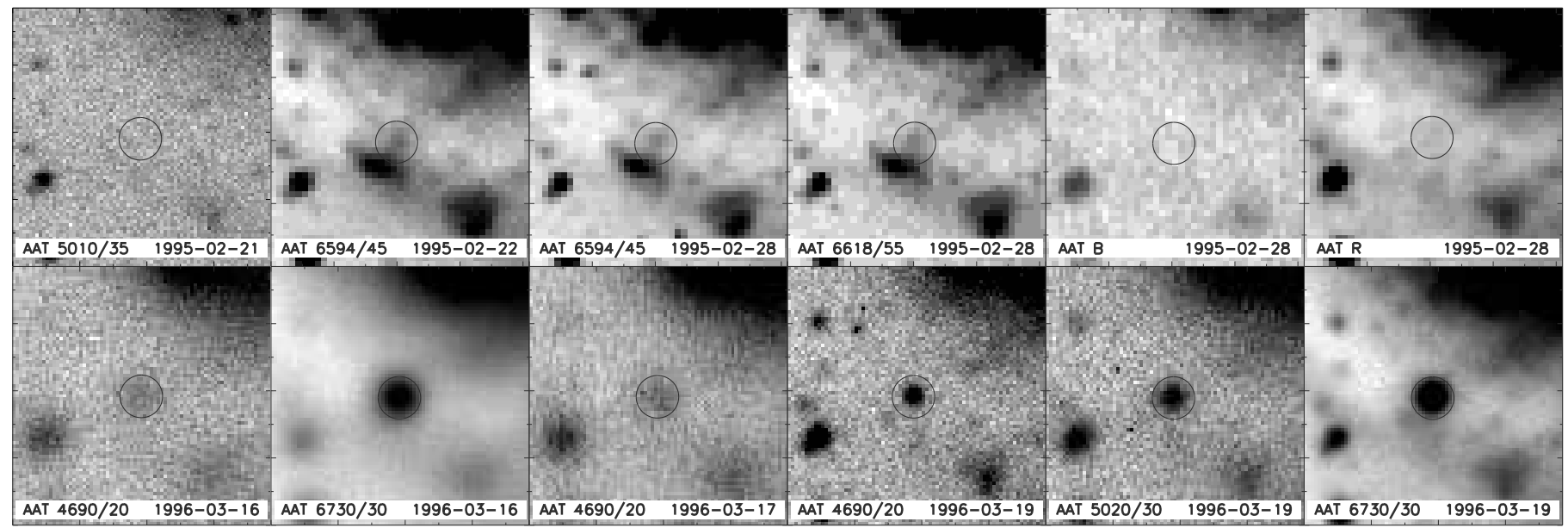

FIG. 4.- Postage-stamp images centered on SN 1996cr from AAT imaging observations. The top six images from 1995-02-21 to 1995-02-28 represent the comparison epoch, while the bottom six images from 1996-03-16 to 1996-03-19 represent the discovery epoch. Despite the poor quality of some images, it is clear that SN 1996cr is consistently luminous in all discovery images spanning several days. See Fig. 2 caption for details of image properties.

TABLE 1

ESO OBSERVATIONS

\begin{tabular}{|c|c|c|c|c|c|}
\hline Instrument & Filter & Date & Exp. & Seeing & Magnitude \\
\hline SUSI/NTT & ESO\#369 ([O III]) & 1993-04-09 & 900 & $0 . ! 7$ & $>21.7$ \\
\hline SUSI/NTT & ESO\#430 (5108 Å cont.) & 1993-04-09 & 900 & $0 . ! 7$ & - \\
\hline SUSI/NTT & ESO\#629 (H $\alpha+[\mathrm{N} \mathrm{II}])$ & 1993-04-09 & 480 & $0 . \prime 7$ & $19.1 \pm 0.2^{\dagger}$ \\
\hline SUSI/NTT & ESO\#700 ([S II]) & 1993-04-09 & 900 & $0 .{ }^{\prime \prime} 7$ & $21.2 \pm 0.3^{\dagger}$ \\
\hline SUSI/NTT & ESO\#443 (7027 A cont.) & 1993-04-09 & 900 & $0 . \prime 7$ & - \\
\hline SUSI/NTT & ESO\#415 ([Fe XI] $\lambda 7892)$ & 1993-04-09 & 900 & $0 . \prime 7$ & - \\
\hline IRAC2/ESO-MPI 2.2m & $\mathrm{J}(1.25 \mu \mathrm{m})$ & $1993-06-25$ & 360 & $0 . \prime 9$ & $>22.0$ \\
\hline IRAC2/ESO-MPI $2.2 \mathrm{~m}$ & $\mathrm{H}(1.65 \mu \mathrm{m})$ & $1993-06-25$ & 360 & $0 . \prime 9$ & $>19.8$ \\
\hline
\end{tabular}

Note. - Column 1: Instrument and telescope. Column 2: Filter. Column 3: UT date of observation given as year-month-day. Column 4: Exposure time in seconds. Column 5: Seeing. Column 6: Aperture-corrected Vega magnitude or $3 \sigma$ upper limit. Details are given in $\S 2.2 .1$. The detected magnitudes denoted by $\dagger$ s provide constraints on line emission from the H II region which spatially overlaps SN 1996cr.

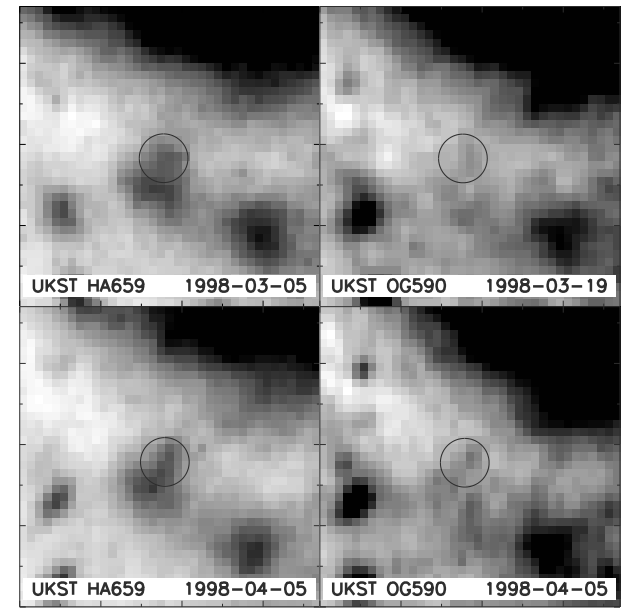

FIG. 5.- Postage-stamp images centered on SN 1996cr from UKST imaging observations in 1998. SN 1996cr is still evident as a slight excess in the $\mathrm{H} \alpha$ images compared to similar narrow-band images in Figs. 2 and 4. See Fig. 2 caption for details of image properties.
The Circinus Galaxy was observed on numerous occasions with the UKST as detailed in Table 4. The data were retrieved from the SUPERCOSMOS archive, ${ }^{15}$ although only a subset of the photographic plates have been properly digitized and archived in a usable form. Fig. 5 shows four images taken in 1998 March and April. The H $\alpha$ images from 1998 (as well as similar degraded ones from 1999 and 2001) show a slight enhancement over the $1995 \mathrm{H} \alpha$ reference images from the AAT and ESO. For the four images that were taken after the discovery window of SN 1996cr and properly digitized, we performed photometry using a 1 .'0 radius circular aperture and previously established zero points. An aperture correction of 0.2 mags was estimated empirically using several bright, isolated point sources in the images. The resulting aperturecorrected magnitudes are presented in Table 4.

\subsubsection{Swift UVOT}

The Circinus Galaxy was observed on 2007-03-23 with the Swift Ultraviolet/Optical Telescope (UVOT) for 617-2461 s depending on the filter. The images were reduced using standard pipeline procedures and photometry was performed on the images using a 3!'0 radius circular aperture. SN 1996cr was not detected in any of the six UVOT filters, with aperture- 
TABLE 2

HST OBSERVATIONS

\begin{tabular}{lccrl}
\hline \hline Instrument & Filter & Date & Exp. & Magnitude \\
\hline WFPC2 & F606W & $1996-08-11$ & 200,400 & - \\
NIC3 & F164N & $1998-03-16$ & 64 & $20.75 \pm 0.28$ \\
NIC3 & F166N & $1998-03-16$ & 64 & $>21.09$ \\
NIC3 & F196N & $1998-03-16$ & 80 & $21.04 \pm 0.29$ \\
NIC3 & F200N & $1998-03-16$ & 80 & $20.59 \pm 0.27$ \\
NIC3 & F212N & $1998-10-16$ & 160 & $20.90 \pm 0.18$ \\
NIC3 & F215N & $1998-10-16$ & 160 & $20.60 \pm 0.19$ \\
WFPC2 & F502N & $1999-04-10$ & 900,900 & $20.67 \pm 0.06$ \\
WFPC2 & F547M & $1999-04-10$ & 60 & $22.81 \pm 0.12$ \\
WFPC2 & F656N & $1999-04-10$ & 800,800 & $17.98 \pm 0.11$ \\
WFPC2 & F814W & $1999-04-10$ & 40 & $21.20 \pm 0.09$ \\
& & & & \\
\hline
\end{tabular}

NOTE. - Column 1: HST instrument and filter. Filter denotes the central filter wavelength in nanometers and the filter width ( $N=$ narrow, $M=$ medium, $\mathrm{W}=$ wide). Details on individual filters can be found in the $\mathrm{WFPC}^{a}$ and NICMOS $^{b}$ Instrument Handbooks. Column 2: UT date of observation given as year-month-day. Column 3: Exposure time(s) in seconds. Column 4: Aperture-corrected Vega magnitude or $3 \sigma$ upper limit. Magnitudes were measured with circular apertures of radius $0 .{ }^{\prime \prime} 2$ and $0 .{ }^{\prime \prime} 5$ for WFPC 2 and NIC3, respectively, and corrected for missing flux due to the shape of the PSF. Note that increasing the aperture radius in the $H S T$ F $656 \mathrm{~N}$ band to $1 .{ }^{\prime \prime} 2$ yields a magnitude of 17.5 (i.e., an increase of $\approx 25 \%$ over the point-like magnitude from SN 1996cr alone) which we attribute to the flux of the underlying $\mathrm{H}$ II region. We have added a systematic error of 0.1 magnitude to the F656N value to reflect the accuracy to which we can deblend the point source emission from the underlying $\mathrm{H}$ II region. The $\mathrm{H}$ II region alone should have a magnitude of $18.7 \pm 0.2$, which is consistent with values measured from our early-time comparison images. Likewise, increasing the aperture in the NIC3 observations any further will begin to include nearby contaminating point sources, as shown in Fig. 3. Finally, we have incorporated an additional systematic error of $0.1 \mathrm{mag}$ to account for the intrapixel sensitivity variations in the NIC3 observations.

${ }^{a} \mathrm{http}: / / \mathrm{www} . s t s c i . e d u /$ instruments/wfpc2/Wfpc2_hand_current/

${ }^{b}$ http://www.stsci.edu/hst/nicmos/documents/handbooks/v4.1/

TABLE 3

AAT TAURUS OBSERVATIONS

\begin{tabular}{|c|c|c|c|c|}
\hline Filter & Date & Exp. & Seeing & Magnitude \\
\hline $5020 / 30\left(\theta_{f}=0\right)$ & $1995-02-21$ & 300 & $1 . \prime 2$ & $>20.0$ \\
\hline $6583 / 45\left(\theta_{f}=9\right)$ & $1995-02-22$ & 300 & $1 . \prime 2$ & $>18.3$ \\
\hline $6583 / 45\left(\theta_{f}=9\right)$ & $1995-02-28$ & 267 & $1 .^{\prime \prime} 1$ & $>18.1$ \\
\hline $6618 / 55\left(\theta_{f}=7\right)$ & $1995-02-28$ & 1442 & $1^{\prime \prime} 1$ & $>18.4$ \\
\hline$R$ & $1995-02-28$ & 60 & $1 . ' 2$ & $>19.6$ \\
\hline$B$ & $1995-02-28$ & 20 & $1 . " 4$ & $>22.1$ \\
\hline $4690 / 26\left(\theta_{f}=5\right)$ & 1996-03-16 & 1200 & $33^{\prime \prime} 5$ & $19.5 \pm 0.6$ \\
\hline $6730 / 30\left(\theta_{f}=5\right)$ & 1996-03-16 & 1200 & $3 .^{\prime \prime} 0$ & $15.8 \pm 0.5$ \\
\hline $4690 / 26$ Z-CUBE $\left(\theta_{f}=5\right)$ & $1996-03-16$ & & $3 !^{\prime \prime} 0$ & \\
\hline $4690 / 26\left(\theta_{f}=0\right)$ & 1996-03-17 & 1200 & $3 .^{\prime \prime} 1$ & $19.8 \pm 0.5$ \\
\hline $4690 / 26\left(\theta_{f}=0\right)$ & 1996-03-19 & 1000 & $1 . " 2$ & $19.7 \pm 0.3$ \\
\hline $5020 / 30\left(\theta_{f}=0\right)$ & 1996-03-19 & 120 & $1 !^{\prime \prime} 3$ & $18.7 \pm 0.4$ \\
\hline $6730 / 30\left(\theta_{f}=0\right)$ & 1996-03-19 & 1000 & $1 . " 3$ & $15.4 \pm 0.3$ \\
\hline
\end{tabular}

Note. - Column 1: Filter, given here as central wavelength and bandwidth in Angstroms. 6370/30, 6583/45, 5020/30, 4690/26 are narrow-band filters used with the AAT TAURUS Tunable Filter (see AAT Filter Catalog ${ }^{a}$ for details), while $B$ and $R$ are standard Johnson filters. Note that for the TAURUS instrument, we also list a tilt angle, which tunes the wavelength range such that $\lambda_{\text {eff }} \approx \lambda_{\text {nom }}\left(1-\theta_{f}^{2} / 28984\right)$, where $\theta_{f}$ is tilt angle in degrees. Column 2: UT date of observation given as yearmonth-day. Column 3: Exposure time in seconds. Column 4: Seeing. Column 5: Aperture-corrected Vega magnitude or $3 \sigma$ upper limit. Details are given in $\S 2.2 .3$.

${ }^{a}$ http://www.aao.gov.au/local/www/cgt/ccdimguide/filtercat.html

corrected $3 \sigma$ upper limits of $V>19.0, B>20.2, U>20.0$, $U V W 1>20.4, U V W 2>20.8$, and $U V M 2>21.2$.

\subsubsection{SNe Optical Monitoring}

There are three additional useful upper-limit constraints based on the SNe monitoring observations of the Circinus
TABLE 4

UKST OBSERVATIONS

\begin{tabular}{cccrll}
\hline \hline Plate \# & Filter & Date & Exp. & Grade & Magnitude \\
\hline OR14405 & IIIaF/OG590 & $1991-07-17$ & 2400 & AI3* & - \\
OR16161 & IIIaF/OG590 & $1994-06-27$ & 2480 & BI3* & - \\
OR16238 & IIIaF/OG590 & $1994-08-12$ & 3300 & AID2 & - \\
OR17455 & IIIaF/OG590 & $1997-03-11$ & 300 & aI* & - \\
OR17484 & IIIaF/OG590 & $1997-03-31$ & 300 & $a^{*}$ & - \\
HA17930 & $4415 / H A 659$ & $1998-03-05$ & 10800 & A2 & $17.1 \pm 0.6$ \\
OR17954 & $4415 /$ OG590 & $1998-03-19$ & 900 & a & $18.3 \pm 0.9$ \\
HA17987 & $4415 / H A 659$ & $1998-04-05$ & 10800 & AT2 & $17.3 \pm 0.4$ \\
OR17988 & $4415 /$ OG590 & $1998-04-05$ & 900 & a & $18.0 \pm 0.7$ \\
OR18316 & $4415 /$ OG590 & $1999-03-11$ & 900 & $\mathrm{a}^{*}$ & - \\
HA18323 & 4415/HA659 & $1999-03-12$ & 2160 & bU* & - \\
HA18328 & 4415/HA659 & $1999-03-14$ & 10800 & bT** & - \\
HA19197 & 4415/HA659 & $2001-05-24$ & 8400 & AU2* & -
\end{tabular}

Note. - Column 1: A one- or two-character code for the filter/emulsion combination along with a running number for all UKST plates. Column 2: Emulsion and filter. OG590 is red, while HA659 is H $\alpha$. Column 3: UT date of observation given as year-month-day. Column 4: Exposure time in seconds. Column 5: All plates are quality controlled and assigned a grade. The first letter of the grade (usually A,B,C) indicates the overall quality of the plate. The subsequent letters indicate specific defects as follows: I - Denotes image size larger than 40 microns. T - Denotes detectable image elongation on most images. $\mathrm{U}$ - Denotes underexposure (relative to exposure time). D - Denotes overexposed plate (high central density). Survey plates are normally also given a grade number; the lower the number the better the plate quality. An 'A' grade plate scores 3 or lower. Plates denoted by '*' were only available as lower quality "Finder" (GIF) format and thus are not as photometrically reliable. Additionally, the spatial resolution of plate OR16238 was too poor to provide a useful comparison magnitude due to source blending. Column 7: Aperture-corrected Vega magnitude. Details are given in $\S 2.2 .4$.

Galaxy by Rev. Robert Evans on 1995-03-31, 1995-08-13, and 1996-03-03 (private communication, 2000). We have adopted upper limits of $V>14$ based on R. Evans' estimated limiting magnitude of $V \approx 15$.

\subsection{Archival X-ray Data}

Details of the various X-ray observations are given in Table 5. We describe below our reduction methods for each dataset. When possible, the X-ray fluxes and absorptioncorrected luminosities for SN 1996cr were calculated via spectral analysis in XSPEC (Arnaud 1996) using the Cash statistic (Cash 1979). Unless stated otherwise, errors on spectral parameters are for $68 \%$ confidence, assuming one parameter of interest. Following the treatment of SN 1987A (e.g., Park et al. 2005), we characterized the X-ray spectra of SN 1996cr with an absorbed variable-abundance nonequilibrium ionization (NEI) shock model (vpshock using NEI version 2.0 in XSPEC) based on ATOMDB (Smith et al. 2001). ${ }^{16}$ The best-fitted parameters to the combined XMMNewton and Chandra HETGS 0th order dataset were as follows: $N_{\mathrm{H}}=8.4 \times 10^{21} \mathrm{~cm}^{-2}, k T=13.4 \mathrm{keV}, Z_{\mathrm{Si}}=2.25 Z_{\odot, \mathrm{Si}}$, $Z_{\mathrm{S}}=3.5 Z_{\odot, \mathrm{S}}, Z_{\mathrm{Ca}}=8 Z_{\odot, \mathrm{Ca}}$, and $Z_{\mathrm{Fe}}=2 Z_{\odot, \mathrm{Fe}} . \quad$ We note, however, that these specific abundances, and even the estimated temperature to some extent, should be used with caution, as there are known to be significant deviations between adopted models (e.g., Nymark et al. 2006). The typical column density, $N_{\mathrm{H}}$, derived from spectral fits to the data is much larger than the estimated Galactic absorption column $\left(\approx 3 \times 10^{21} \mathrm{~cm}^{-2}\right)$, implying significant internal absorption either from the disk of the Circinus Galaxy at large or the immediate vicinity of SN $1996 \mathrm{cr}$. Fluxes were measured by varying the normalization of the above model in the $0.5-2 \mathrm{keV}$ or

16 While this model is believed to best characterize the X-ray emission from young SNe such as SN 1996cr, there are still many serious caveats. See http://cxc.harvard.edu/atomdb/issues_caveats.html for details. 
TABLE 5

X-RAY OBSERVATIONS

\begin{tabular}{lcrrrrrl}
\hline \hline \multicolumn{1}{c}{ Instrument } & \multicolumn{1}{c}{ Date } & Exp. & $F_{0.5-2 \mathrm{keV}}$ & $F_{2-8 \mathrm{keV}}$ & $F_{0.5-2 \mathrm{keV}}^{c}$ & $F_{2-8 \mathrm{keV}}^{c}$ & Comment \\
\hline ASCA & $1995-02-14$ & 61.0 & $<5^{\dagger}$ & $<142^{\dagger}$ & $<19^{\dagger}$ & $<152^{\dagger}$ & \\
ROSAT HRI & $1995-09-14$ & 4.1 & $<6$ & - & $<23$ & - & \\
ROSAT HRI & $1996-02-18$ & 1.1 & $<23$ & - & $<93$ & - & \\
ROSAT HRI & $1996-09-13$ & 1.8 & $<11$ & - & $<44$ & - & \\
ROSAT HRI & $1997-03-03$ & 26.4 & $<2$ & - & $<8$ & - & \\
ROSAT HRI & $1997-08-17$ & 45.9 & $<1$ & - & $<5$ & - & \\
BeppoSAX & $1998-03-13$ & 85.2 & $<14^{\dagger}$ & $<154^{\dagger}$ & $<51^{\dagger}$ & $<165^{\dagger}$ & \\
Chandra ACIS-S & $2000-01-16$ & 1.0 & $13 \pm 3$ & $142 \pm 35$ & $62 \pm 16$ & $142 \pm 35$ & $21 \%$ pile-up \\
Chandra ACIS-S & $2000-03-14$ & 4.9 & $14 \pm 3$ & $133 \pm 15$ & $51 \pm 10$ & $140 \pm 16$ & $2 \%$ pile-up \\
Chandra ACIS-S & $2000-03-14$ & 23.1 & $10 \pm 2$ & $120 \pm 20$ & $45 \pm 9$ & $120 \pm 20$ & $20 \%$ pile-up \\
Chandra HETGS & $2000-06-15$ & 67.1 & $12 \pm 2$ & $108 \pm 9$ & $43 \pm 9$ & $118 \pm 9$ & $3 \%$ pile-up \\
BeppoSAX & $2001-01-07$ & 27.2 & $<80^{\dagger}$ & $<614^{\dagger}$ & $<292^{\dagger}$ & $<620^{\dagger}$ & \\
Chandra ACIS-S & $2001-05-02$ & 4.4 & $12 \pm 2$ & $136 \pm 20$ & $53 \pm 11$ & $136 \pm 20$ & $21 \%$ pile-up \\
XMM-Newton & $2001-08-06$ & 85.5 & $16 \pm 1$ & $144 \pm 5$ & $61 \pm 2$ & $154 \pm 5$ & \\
Chandra HETGS & $2004-06-02$ & 55.0 & $23 \pm 3$ & $186 \pm 11$ & $88 \pm 12$ & $204 \pm 11$ & $3 \%$ pile-up \\
Chandra HETGS & $2004-11-28$ & 59.0 & $24 \pm 3$ & $196 \pm 12$ & $93 \pm 11$ & $215 \pm 13$ & $3 \%$ pile-up \\
Swift XRT & $2007-03-25$ & 8.0 & $35 \pm 8$ & $206 \pm 39$ & $124 \pm 28$ & $222 \pm 42$ & \\
& & & & & & &
\end{tabular}

Note. - Column 1: Satellite and instrument. Column 2: Starting date of observation. Column 3: Exposure time in ksec. Columns 4 and 5: Flux in the $0.5-2 \mathrm{keV}$ and $2-8 \mathrm{keV}$ bands respectively, determined from the best-fit model in XSPEC in units of $10^{-14} \mathrm{erg} \mathrm{s}^{-1} \mathrm{~cm}^{-2}$. For upper limits, we have adopted the spectrum from the closest Chandra observation. For instruments unable to resolve SN $1996 \mathrm{cr}$ from the bright nucleus of the Circinus Galaxy, denoted by ${ }^{\dagger}$, upper limit fluxes were estimated by subtracting the total flux of the Circinus Galaxy without SN 1996cr from the total unresolved flux (see $\S 2.3$ for details and caveats). Columns 6 and 7 : Absorption-corrected flux in the $0.5-2 \mathrm{keV}$ and $2-8 \mathrm{keV}$ bands respectively, determined from the best-fit model in XSPEC in units of $10^{-14} \mathrm{erg} \mathrm{s}^{-1} \mathrm{~cm}^{-2}$. Column 8: Comments.

$2-8 \mathrm{keV}$ bands. Our adopted model fit all of the spectral data adequately, although we note that there was typically some excess residual emission around prominent emission lines, as well as an apparent $\sim 2 \sigma$ soft excess in the 2004 HETGS data. These issues have a negligible effect, however, on our flux estimates to within errors and will be addressed in more detail in a separate publication on X-ray spectral analysis of SN 1996cr (F. Bauer et al., in preparation).

\subsubsection{Chandra}

The Circinus Galaxy was observed on several occasions with Chandra using the backside-illuminated Advanced Imaging CCD Spectrometer (ACIS-S) in the focal plane, both with and without the High-Energy Transmission Grating Spectrometer (HETGS). Archival ACIS-S and HETGS zeroth-order data were retrieved from the Chandra Data Archive and processed following standard procedures using CIAO (v3.4) software. Additionally we removed the 0.'5 pixel randomization, corrected for charge transfer inefficiency (CTI), performed standard ASCA grade selection, excluded bad pixels and columns, and screened for intervals of excessively high background (none was found). Analysis was performed on reprocessed Chandra data, primarily using CIAO, but also with FTOOLS (v6.3) and custom software including ACIS EXTRACT (v3.128; Broos et al. 2005). Spectra were extracted using ACIS EXTRACT with a 95\% encircled-energy region derived from the Chandra PSF library. As SN 1996cr lies at the edge of diffuse emission associated with the AGN, a background spectrum was extracted from a local annular region after excluding nearby point sources and the strongest portions of the circumnuclear halo and ionization cone associated with the AGN (the annulus size was grown until approximately 100 counts could be extracted). We generated calibration products (including an aperture correction) for the HETGS/ACIS-S spectra and fit them separately within XSPEC using our best-fit model to estimate the flux. The majority of the observations were performed in configurations which mitigate pile-up; when significant we have noted the estimated pile-up fractions for SN $1996 \mathrm{cr}$, and corrected for them using the XSPEC pileup model of Davis (2001).

\subsubsection{XMM-Newton}

The Circinus Galaxy was observed once with XMM-Newton using the EPIC p-n and MOS1/MOS2 detectors on 2001-0806 for $\approx 104$ ks. Archival data were retrieved from the $X M M$ Newton Science Archive and processed following standard procedures with SAS (v7.0.0). Additionally, we screened for and removed intervals of excessively high background $(\approx$ $15 \%$ for MOS1/MOS2 and $\approx 40 \%$ for $\mathrm{p}-\mathrm{n}$ ), leaving $85.5 \mathrm{ks}$, $91.8 \mathrm{ks}$, and $59.5 \mathrm{ks}$ of useful exposure with the MOS1, MOS2, and p-n instruments, respectively. Spectra were extracted with SAS using a circular aperture of radius $11 !^{\prime \prime} 0$ and local background was extracted in a manner similar to that done for the Chandra observations. We generated calibration products for the p-n/MOS1/MOS2 datasets and joint-fit the three spectra within XSPEC using our best-fit model to estimate the flux.

Additionally, we used the XMM-Newton data to place upper limits on the potential flux from SN 1996cr in the ASCA and BeppoSAX datasets, both of which lack the spatial resolution to resolve the galaxy into its various components (e.g., see Fig. 1 of Bauer et al. 2001). To this end, spectra for the entire Circinus Galaxy were extracted from a 2!5 radius circular aperture, excluding emission from SN 1996cr. Unfortunately, the ultraluminous X-ray source CG X-1 is known to vary with a period of $7.5 \mathrm{hr}$ and comprises a non-negligible fraction of the total X-ray emission from Circinus. Thus we extracted a pessimistic spectrum additionally excluding CG X-1. This provides conservative upper limits for the total emission from the Circinus Galaxy during the ASCA and BeppoSAX observations, under the assumption that the fluxes of the various emission components within the Circinus Galaxy (aside from CG X-1 and SN 1996cr) have not changed since 1995. Based on the spatially resolved observations that we do have, this assumption appears reasonable. More optimistic upper limits can be estimated by further subtracting the time-averaged 
flux from CG X-1. Given ambiguities in determining how the unresolved observations sample the light curve of CG X-1, we simply provide here the flux of CG X-1 in its "high" state (see Bauer et al. 2001) which is $5.7 \times 10^{-13}$ ergs s${ }^{-1} \mathrm{~cm}^{-2}$ and $4.3 \times 10^{-12} \mathrm{ergs} \mathrm{s}^{-1} \mathrm{~cm}^{-2}$ in the $0.5-2 \mathrm{keV}$ and $2-8 \mathrm{keV}$ bands, respectively, and note that optimistic upper limits for the flux of SN $1996 \mathrm{cr}$ would likely be a factor of $\approx 2-3$ lower.

\subsubsection{ROSAT}

The Circinus Galaxy was observed on several occasions with ROSAT using the High-Resolution Imager (HRI). Processed data were retrieved from the High Energy Astrophysics Science Archive Research Center (HEASARC), and analysis was performed using FTOOLS and custom software. We adopted the best-fit model from the combined observations (see above) to estimate the ROSAT HRI upper limits. Upper limits were measured using a $10^{\prime \prime}$ aperture and a local background extracted to match roughly the amount of diffuse and scattered emission thought to reside within the source aperture. Count rates were converted to fluxes with XSPEC using the final ROSAT calibration products and our adopted best-fit model. Adopting similar models does not have a significant impact on our derived flux upper limits.

\subsubsection{Swift}

The Circinus Galaxy was observed once with the Swift X-ray Telescope (XRT) on 2007 March 23 for 7.4 ks. Processed data were retrieved from Swift Archive, and analysis was performed using FTOOLS and custom software. SN 1996cr is visibly separated from the nucleus $\left(25^{\prime \prime}\right.$ separation) and diffuse circumnuclear emission and appears to be only marginally contaminated. A spectrum of SN 1996cr was extracted from the event list using a 10 pixel $(23 . ' 5)$ radius circular aperture, masked to exclude emission from the nucleus and diffuse halo to as large an extent as possible. Additionally, a background was extracted in a manner similar to that done for the Chandra observations so as to match roughly the amount of diffuse and scattered emission believed to be present within the source aperture. Low photon statistics, however, limited the fidelity of the background subtraction. We generated calibration products for the spectrum and fit it within XSPEC using our best-fit model to estimate the flux.

\subsubsection{ASCA}

The Circinus Galaxy was observed once with ASCA using the Gas Imaging Spectrometers (GIS) and Solid State Imaging Spectrometers (SIS) on 1995-02-14 for 61.1 ks. Processed data were retrieved from HEASARC, providing $\approx 23-36 \mathrm{ks}$ of usable data. Analysis was performed using FTOOLS and custom software. The target appears unresolved with $A S C A$ and was treated as a point source for extraction purposes. Spectra for the entire Circinus Galaxy were extracted from a 2.5 radius circular aperture, while backgrounds were taken from blank-sky observations at the same position and with identical screening criteria. Contamination from a neighboring source $5^{\prime}$ away was minimal. Total fluxes were measured via simultaneous spectral fitting of the GIS and SIS data and upper limits for SN 1996cr were estimated by subtracting the flux estimated from the XMM-Newton data as detailed above.

\subsubsection{BeppoSAX}

The Circinus Galaxy was observed on two occasions with BeppoSAX using the Low Energy Concentrator Spectrometer (LECS) and Medium Energy Concentrator Spectrometer (MECS) on 1998-03-03 for 83.9/138.0 ks and on 200101-07 for 26.9/52.1 ks. Processed data were retrieved from HEASARC, providing 83.7/137.2 ks and 26.8/51.7 ks of usable data, respectively. Analysis was performed using FTOOLS and custom software. The target appears unresolved with BeppoSAX and was treated as a point source for extraction purposes. LECS and MECS spectra for the entire Circinus Galaxy were extracted from a $3-4^{\prime}$ radius circular apertures (limited by the availability of calibration files), while backgrounds were taken from blank-sky observations at the same position and with identical screening criteria. Contamination from a neighboring source $5^{\prime}$ away was minimal. Total fluxes were measured via simultaneous spectral fitting of the LECS and MECS data, and upper limits for SN 1996cr were estimated by subtracting the flux estimated from the $X M M$ Newton data as detailed above.

\subsection{Archival and Proposed Radio Data \\ 2.4.1. Australia Telescope Compact Array}

Radio observations of the Circinus Galaxy spanning 1995 to 2006 were retrieved from the Australia Telescope Compact Array (ATCA) archive ${ }^{17}$ and are presented in Table 6 and Fig. 6. The data were reduced with MIRIAD (v4.0.5) following the procedures outlined in the ATNF Miriad User Manual. ${ }^{18}$

The "primary" flux calibrator is 1934-638, which is assumed to be constant in time with flux densities of 14.94, $11.60,5.83,2.84$, and $1.03 \mathrm{Jy}$ at $20,13,6,3,1 \mathrm{~cm}$, respectively. As noted in Table 6, there are a few instances in which 1938-638 is not included as part of the dataset, although the observations are initially "primed" using this source; for such cases, fluxes of the "secondary" calibrators were compared to their interpolated historical values from calibration monitoring efforts ${ }^{19}$ and were fixed to those values when they strayed by more than $\sim 20 \%$ from them. The secondary calibrators, which served as the gain, bandpass, and phase calibrators for the Circinus Galaxy, varied from observation to observation and include sources 1921-293 (bandpass, alternate $1 \mathrm{~cm}$ flux), 1329-665 (bandpass), 1549-790 (bandpass), 1414-59 (phase), and 1236-684 (phase). Both source and calibration data were flagged to remove bad time intervals and channels, time intervals strongly affected by interference, and obvious emission and absorption lines. The calibration tables were applied to the Circinus Galaxy data, from which deconvolved, primary-beam-corrected images were made. On rare occasions, a phase self-calibration was required. Integrated flux densities (determined from IMFIT) or $3 \sigma$ upper limits (determined from IMSTAT) were then determined for SN 1996cr. These values are provided in Table 6 . There appears to be considerable dispersion in $22.5 \mathrm{GHz}$ flux densities, which were mostly acquired as part of larger Australia Long Baseline Array observations. We lack comparison data at other wavelengths to determine if this is simply additional calibration uncertainty or potentially real flux variations. However, given that there appears to be dispersion even between observations taken simultaneously, we caution that the dispersion is not intrinsic, but rather due to systematic errors.

\footnotetext{
$17 \mathrm{http} / / /$ atoa.atnf.csiro.au/

$18 \mathrm{http} / / / \mathrm{www}$. atnf.csiro.au/computing/software/miriad/

$19 \mathrm{http}: / /$ www.narrabri.atnf.csiro.au/calibrators/
} 
TABLE 6

ATCA RADIO OBSERVATIONS

\begin{tabular}{|c|c|c|c|c|c|c|c|c|c|c|c|c|c|}
\hline \multirow[t]{2}{*}{ Obs. Date } & \multirow[t]{2}{*}{ Obs. ID } & \multirow[t]{2}{*}{ Array } & \multicolumn{2}{|c|}{ K-Band } & \multicolumn{2}{|c|}{$X$-Band } & \multicolumn{2}{|c|}{ C-Band } & \multicolumn{2}{|c|}{ S-Band } & \multicolumn{2}{|c|}{ L-Band } & \multirow[t]{2}{*}{ Comments } \\
\hline & & & $\nu$ & $S_{\nu}$ & $\nu$ & $S_{\nu}$ & $\nu$ & $S_{\nu}$ & $\nu$ & $S_{\nu}$ & $\nu$ & $S_{\nu}$ & \\
\hline 1995-03-03 & $\mathrm{C} 204$ & 375 & - & - & 8640 & $>0.7$ & 4800 & $>1.3$ & - & - & - & - & mosaic field "a" \\
\hline 1995-03-03 & C204 & 375 & - & - & 8640 & $>0.7$ & 4800 & $>0.9$ & - & - & - & - & mosaic field "b" \\
\hline 1995-03-30 & C363 & $1.5 \mathrm{~A}$ & - & - & - & - & - & - & - & - & 1418 & $>6.6$ & \\
\hline 1995-04-16 & C418 & $6 \mathrm{C}$ & - & - & - & - & - & - & 2768 & $>1.1$ & 1418 & $>1.2$ & \\
\hline $1995-05-27$ & C204 & 375 & - & - & 8640 & $>0.7$ & 4800 & $>1.3$ & - & - & - & - & mosaic field "a" \\
\hline $1995-05-27$ & C204 & 375 & - & - & 8640 & $>0.7$ & 4800 & $>0.6$ & - & - & - & - & mosaic field "b" \\
\hline 1995-06-07 & C204 & 375 & - & - & 8640 & $>0.7$ & 4800 & $>1.5$ & - & - & - & - & mosaic field "a" \\
\hline 1995-06-07 & C204 & 375 & - & - & 8640 & $>1.5$ & 4800 & $>0.5$ & - & - & - & - & mosaic field "b" \\
\hline $1995-07-27$ & C204 & $6 \mathrm{C}$ & - & - & - & - & - & - & 2368 & $>1.3$ & 1376 & $>3.4$ & \\
\hline 1995-08-01 & СТ09 & $750 \mathrm{~B}$ & - & - & - & - & - & - & - & - & 1418 & $>17$ & \\
\hline 1996-02-06 & C466 & $750 \mathrm{D}$ & - & - & - & - & - & - & - & - & 1664 & $>1.5$ & \\
\hline 1996-08-01 & C363 & 375 & - & - & 8640 & $0.5 \pm 0.1$ & 4800 & $>1.2$ & - & - & - & - & \\
\hline $1996-12-15$ & C586 & 375 & - & - & 8512 & $2.2 \pm 0.3$ & 4928 & $1.8 \pm 0.2$ & - & - & - & - & mosaic field "a" \\
\hline $1996-12-15$ & C586 & 375 & - & - & 8512 & $2.0 \pm 0.2$ & 4928 & $1.8 \pm 0.2$ & - & - & - & - & mosaic field "b" \\
\hline 1996-12-05 & C505 & 375 & - & - & - & - & - & - & 2368 & $>0.4$ & 1384 & $>0.7$ & \\
\hline 1997-06-17 & V100 & $6 \mathrm{~A}$ & - & - & 8425 & $14 \pm 1$ & - & - & - & - & - & - & \\
\hline 1997-06-17 & V100 & $6 \mathrm{~A}$ & - & - & 8425 & $15 \pm 3$ & - & - & - & - & - & - & spectral-line mode \\
\hline 1997-12-05 & V099 & $6 \mathrm{C}$ & - & - & - & - & - & - & 2268 & $22 \pm 3$ & - & - & \\
\hline 1997-12-05 & V099 & $6 \mathrm{C}$ & - & - & - & - & - & - & 2268 & $37 \pm 4$ & - & - & spectral-line mode \\
\hline $1997-12-31$ & C694 & $6 \mathrm{C}$ & - & - & 8500 & $96 \pm 15$ & 4800 & $119 \pm 12$ & - & - & - & - & $\begin{array}{l}8.5 \mathrm{GHz} \text { extrapolated } \\
\text { from } 6.0 \mathrm{GHz} \text { data }\end{array}$ \\
\hline 1999-06-19 & C788 & 375 & - & - & - & - & - & - & - & - & 1384 & $89 \pm 3$ & \\
\hline 1999-06-19 & C788 & 375 & - & - & - & - & - & - & - & - & 1418 & $85 \pm 3$ & spectral-line mode \\
\hline 2000-06-19 & V137 & $6 \mathrm{~B}$ & 22190 & $76 \pm 21$ & - & - & - & - & - & - & - & - & \\
\hline 2000-06-19 & V137 & $6 \mathrm{~B}$ & 22203 & $72 \pm 20$ & - & - & - & - & - & - & - & - & \\
\hline 2000-06-19 & V137 & $6 \mathrm{~B}$ & 22215 & $69 \pm 21$ & - & - & - & - & - & - & - & - & \\
\hline 2002-07-18 & V137 & $1.5 \mathrm{G}$ & 22172 & $63 \pm 10$ & - & - & - & - & - & - & - & - & \\
\hline 2002-07-18 & V137 & $1.5 \mathrm{G}$ & 22216 & $71 \pm 10$ & - & - & - & - & - & - & - & - & \\
\hline 2002-07-18 & V137 & $1.5 \mathrm{G}$ & 22224 & $70 \pm 10$ & - & - & - & - & - & - & - & - & \\
\hline 2003-09-07 & C1224 & EW367 & 23659 & $63 \pm 10$ & - & - & - & - & - & - & - & - & phase cal only, flux forced \\
\hline 2003-11-03 & C1049 & H214 & 16960 & $80 \pm 10$ & - & - & - & - & - & - & - & - & \\
\hline 2003-11-03 & C1049 & H214 & 19008 & $77 \pm 10$ & - & - & - & - & - & - & - & - & \\
\hline 2003-11-04 & C1049 & H214 & 21056 & $79 \pm 10$ & - & - & - & - & - & - & - & - & \\
\hline 2003-11-04 & C1049 & H214 & 22796 & $73 \pm 10$ & - & - & - & - & - & - & - & - & \\
\hline 2003-11-08 & C1049 & $1.5 \mathrm{D}$ & - & - & 8256 & $163 \pm 10$ & 5056 & $227 \pm 11$ & - & - & - & - & \\
\hline 2004-04-02 & $\mathrm{C} 1424$ & $6 \mathrm{~A}$ & - & - & 8640 & $162 \pm 8$ & 4800 & $240 \pm 10$ & 2368 & $364 \pm 16$ & 1384 & $481 \pm 19$ & \\
\hline 2004-05-20 & CX065 & $1.5 \mathrm{~B}$ & 22088 & $63 \pm 15$ & - & - & - & - & - & - & - & - & phase cal only, flux forced \\
\hline 2004-06-13 & V176 & $750 \mathrm{D}$ & 22209 & $57 \pm 13$ & - & - & - & - & - & - & - & - & \\
\hline 2004-06-13 & V176 & $750 \mathrm{D}$ & 22195 & $59 \pm 11$ & - & - & - & - & - & - & - & - & \\
\hline 2004-08-01 & V176B & H168 & 22209 & $66 \pm 6$ & - & - & - & - & - & - & - & - & \\
\hline 2004-08-01 & V176B & H168 & 22195 & $66 \pm 6$ & - & - & - & - & - & - & - & - & \\
\hline 2005-03-14 & $\mathrm{C} 1368$ & $\mathrm{H} 214$ & 22193 & $51 \pm 15$ & - & - & - & - & - & - & - & - & \\
\hline 2005-04-20 & C1368 & $750 \mathrm{~A}$ & 22194 & $68 \pm 6$ & - & - & - & - & - & - & - & - & phase cal only \\
\hline 2005-11-13 & C1049 & $1.5 \mathrm{C}$ & - & - & 8640 & $159 \pm 5$ & 4800 & $246 \pm 12$ & - & - & - & - & \\
\hline 2006-04-29 & C1049 & $\mathrm{H} 214$ & 18752 & $77 \pm 7$ & - & - & - & - & - & - & - & - & \\
\hline 2006-04-29 & C1049 & $\mathrm{H} 214$ & 21056 & $67 \pm 6$ & - & - & - & - & - & - & - & - & \\
\hline 2006-06-21 & C1049 & $1.5 \mathrm{D}$ & - & - & 8640 & $154 \pm 11$ & 4800 & $261 \pm 10$ & - & - & - & - & \\
\hline 2006-09-14 & C1341 & H75 & - & - & - & - & - & - & - & - & 1418 & $505 \pm 20$ & \\
\hline 2007-06-24 & VX013A & - & 22316 & $67 \pm 6$ & - & - & - & - & - & - & - & - & \\
\hline
\end{tabular}

NOTE. - Column 1: Starting date of observation. Column 2: Observing program ID. Column 3: Array configuration. Columns 4, 6, 8, 10, and 12: Mean frequency of observed band in

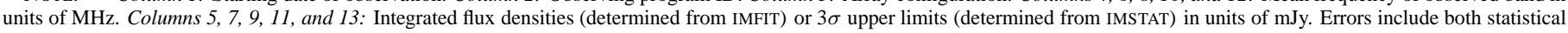

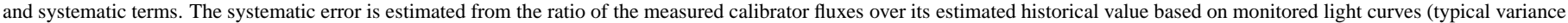
was 5-20\%). Column 14: Comments. Observations taken as mosaics, in spectral-line mode, or with limited calibration sources are duly noted.

We note that the only other strong point source in the field of view was the nucleus of the Circinus Galaxy, which is likely due to emission from the Compton-thick AGN or a compact circumnuclear starburst. As a check on the flux of SN 1996cr, we extracted flux measurements from the pointlike nucleus in a manner identical to SN 1996cr. While the nucleus could vary intrinsically and may additionally be contaminated at long wavelengths by diffuse emission from the extended disk, it still provides us with a secondary estimate of any systematic error associated with a given observation. We find that the majority of measurements of the nucleus lie between 10-30\% of its mean total flux in each band, although a few observations (mainly at $22.5 \mathrm{GHz}$ where contamination from water maser emission is possible) vary by a factor of up to four; we have adopted larger systematic errors for these observations as a result. Thus, aside from the $22.5 \mathrm{GHz}$ data, we are generally confident in our measured fluxes for SN $1996 \mathrm{cr}$.

\subsubsection{Australia Long Baseline Array}

We carried out Very Long Baseline Interferometry (VLBI) observations of SN 1996cr on 2007-06-24 using the following telescopes of the Australian Long Baseline Array: ATCA, Mopra, Parkes, Hobart, and Ceduna. ${ }^{20}$ The observations were phase-referenced to the nearby calibrator PMN J1355-6326. We observed both senses of circular polarization at a frequency of $22 \mathrm{GHz}$ with a total bandwidth of $65 \mathrm{MHz}$, and

\footnotetext{
${ }^{20}$ See http://www.atnf.csiro.au/vlbi/ for details.
} 
for a total time of $\sim 9 \mathrm{~h}$. We report here only our preliminary results and note that a full description is forthcoming $(\mathrm{N}$. Bartel et al., in preparation). SN 1996cr was detected only on the three baselines involving the Parkes, ATCA and Mopra telescopes. The flux of SN 1996cr is consistent with previous ATCA measurements, while its extent appears resolved by the longest baselines at $\gtrsim 3 \sigma$. Although Gaussian and disk models provide adequate fits, we adopt a model consisting of an optically thin spherical shell, as was found to be appropriate for SN 1993J (see, e.g., Bietenholz et al. 2001; Bartel et al. 2002). For a ratio of the outer to the inner shell radius of 1.25 , we find an outer angular radius of $\sim 5$ mas, which corresponds to $\sim 2.8 \times 10^{17} \mathrm{~cm}$ for an assumed distance of $3.8 \mathrm{Mpc}$. Modeling a smaller outer to inner shell ratio like 1.10 could perhaps narrow the angular radius by $\sim 5 \%$, while using a filled-center model (equivalent to an inner shell radius of 0 ) might increase the angular radius by $\sim 20 \%$.

\subsection{Archival Gamma-Ray Burst Data}

Among the numerous GRB-associated SNe that now exist, nearly all have been identified as type Ibc's, which suggests that they arise from Wolf-Rayet progenitors. Although SN 1996cr is identified as a type IIn SN at late times, it is worth searching for any evidence of temporal and spatial gamma-ray emission, as its original type may have been quite different. Additionally, there are at least two events in which a good observational case can be made for the association of a GRB with a type IIn SN: GRB 011121/SN 2001ke (Garnavich et al. 2003) and GRB 970514/SN 1997cy (Germany et al. 2000). We find eight BATSE GRB detections over the lifetime of the Compton Gamma-Ray Observatory (Meegan et al. 1996) which include the Circinus Galaxy within their $3 \sigma$ error regions. One of these GRBs, 4B 960202, appears to lie within the explosion date constraints of SN $1996 \mathrm{cr}$, suggesting a tentative association. Importantly, however, 4B 960202 was detected by multiple Gamma-ray observatories and has a dramatically smaller error estimate via triangulation from the Interplanetary Network (IPN; Hurley et al. 1999, 2005). The full IPN $3 \sigma$ confidence annulus is only $\approx 0.33$ wide and lies $\gtrsim 1.3$ from the $\mathrm{SN}$ at its closest tangential point, thus strongly ruling out any identification with SN $1996 \mathrm{cr}$.

\section{ISOLATION OF SN 1996cr'S EXPLOSION DATE}

Tables 3-6 give a detailed summary of the observations relevant for determining the explosion date of SN 1996cr. While SN 1996cr was discovered in the X-ray band, the archival $\mathrm{X}$-ray data available are too limited and subject to potential geometrical and structural effects (e.g., early absorption, cavities) to narrow down the explosion date to better than an $\sim 10$ yr window between 1990-2000. Although SN 1996cr is relatively well-sampled at radio frequencies, those observations similarly suffer from the same early-time effects, thus limiting their utility to date the SN. Fig. 6 demonstrates that the radio emission begins to sharply "turn on" in mid-1996, signaling that SN $1996 \mathrm{cr}$ must have exploded sometime prior to this. Several narrow-band optical images from the AAT fortuitously bracket the SN explosion date between 1995-0228 and 1996-03-16, allowing us to isolate the SN explosion to within a year. Final confirmation of SN 1996cr comes in the form of the optical spectrum, which signals many telltale features of a SN embedded in dense circumstellar material (CSM).

We note that SN 1996cr's position coincides with a small, powerful H II region. However, it is clear from a comparison of Figs. 2 and 3 that much of the $\mathrm{H} \alpha$ is due to the unresolved SN-CSM interaction ( $\sim 70 \%$; see also Table 2$)$ and thus is likely to be associated with photoionized wind material surrounding the progenitor (e.g., Fesen et al. 1999). Contamination is likely to be negligible for other wavelengths.

The tightest direct temporal constraints arise from comparing the $R$-band image taken on 1995-02-28 with the narrowband [S II] image taken on 1996-03-16. As such, we cannot constrain the spectral form of SN 1996cr at these early times (i.e., whether it is line- or continuum-dominated), which make conversions between various bands somewhat uncertain. For simplicity, we adopt here an intrinsically flat continuum absorbed by $E(B-V)=2.2$ mags at early times. This is likely conservative, considering typical early $\mathrm{SNe}$ are intrinsically quite blue. The extinction was determined using the three narrow-band discovery-image magnitude constraints and is $\approx 0.8$ mags more than a standard conversion of our best-fitted $N_{\mathrm{H}}=8.4 \times 10^{21} \mathrm{~cm}^{-2}$ from the late-time X-ray spectra; a factor of $\sim 2$ variance is commonly seen between extinction and $N_{\mathrm{H}}$ in our own Galaxy (e.g., Burstein \& Heiles 1978), so this discrepancy is probably not necessarily reason for concern, although it could be indicative of further early-time absorption. We note that this model should provide adequate conversions to broad-band magnitudes since early-time SNe spectra are often dominated by strong, broad lines that blend to form a pseudo-continuum.

Using SYNPHOT in IRAF to convert from the narrow AAT He II, [O III], and [S II] bands, we find an equivalent discovery magnitude of $V \approx 17.8$. At the distance of the Circinus Galaxy, this equates to an uncorrected absolute magnitude of $M_{\mathrm{V}} \approx-10.2$, or $M_{\mathrm{Vc}} \lesssim-15$ when corrected for the minimum expected extinction. This provides a strong lower limit since the SN likely went off prior to the discovery images and the apparent extinction estimated from the discovery magnitudes is substantially higher.

\section{CHARACTERIZING SN $1996 \mathrm{CR}$}

The basic observational picture for core-collapse $\mathrm{SNe}$ starts at shock breakout (e.g., Chevalier \& Fransson 1994, hereafter CF94), where the blast wave emerges from the progenitor with a typical velocity of $\gtrsim 10^{4} \mathrm{~km} \mathrm{~s}^{-1}$ and propagates into the CSM formed by the pre-SN stellar wind. The interaction between the ejecta in the forward shock and the CSM leads to the formation of a reverse shock, which travels back into the expanding ejecta — at least relative to the outward expanding contact discontinuity that separates the shocked ejecta and the shocked CSM. Fig. 7 provides a basic one-dimensional picture of the overall scenario. The full three-dimensional structure could be much more complicated. For instance, the contact discontinuity is susceptible to Rayleigh-Taylor instabilities, which are only resolved in multi-dimensions (e.g., Chevalier et al. 1992; Dwarkadas 2000). Likewise, asymmetries could exist in the CSM or SN explosion itself due to the nature of the progenitor or the existence of a binary companion (e.g., SN 1979C, SN 1987A; McCray 1993; Crotts \& Heathcote 2000; Montes et al. 2000; Sugerman et al. 2005). For a typical slow $\left(v_{w} \sim 10 \mathrm{~km} \mathrm{~s}^{-1}\right)$, dense $\left(\dot{M} \sim 10^{-4}-10^{-6} \mathrm{M}_{\odot} \mathrm{yr}^{-1}\right)$ constant progenitor wind, the forward shock/CSM interaction produces a very hot shell of shocked CSM $\left(T \sim 10^{9} \mathrm{~K}\right)$, while the reverse shock/ejecta interaction produces a denser, cooler shell of shocked ejecta $\left(T \sim 10^{7} \mathrm{~K}\right)$. The latter interaction has a much higher emission measure and generates copious far-UV and X-ray emis- 


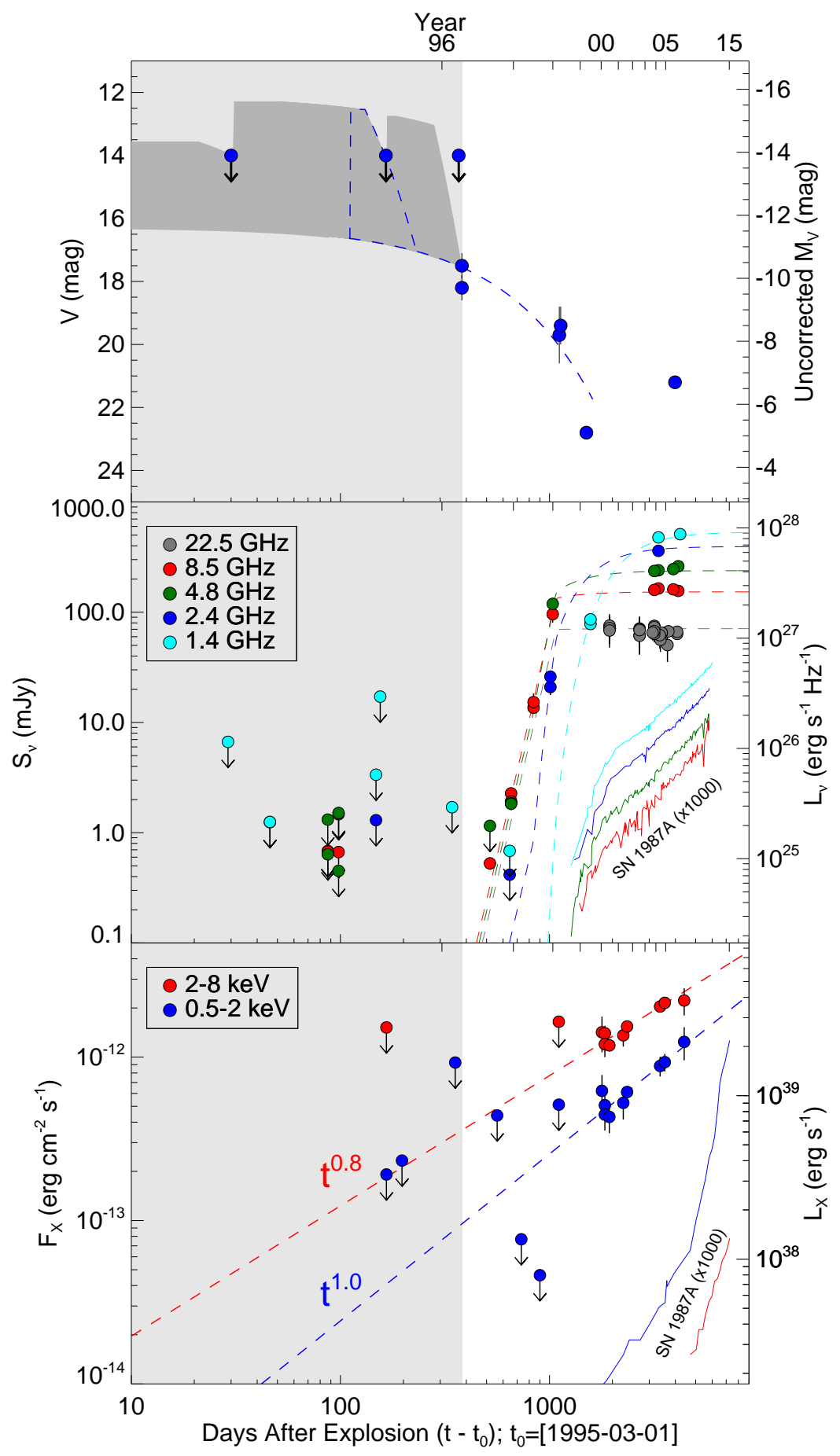

FIG. 6. - The solid circles denote the light curves for $V$-band (top panel), radio (middle panel), and absorption-corrected X-ray (bottom panel) data. Fluxes are shown on the left, while luminosities are on the right. The light grey shaded region on the left denotes the current explosion window. For the $V$-band, we also show a typical $V$-band light curve based on five type In $\mathrm{SNe}$ (dashed blue line). There is a large range in properties at early times, for which we show only the upper and lower extremes expected; SN 1996cr's maximum light could lie anywhere in between. We have normalized to fit the initial discovery magnitudes and adopted a late-time decline rate of $\Delta V=0.0035$ per day to fit the HST data. None of the magnitudes has been corrected for extinction, which is likely to be in excess of $A_{\mathrm{V}} \approx 4.5$. The dark grey region denotes the range of parameter space where our adopted SN light curve is not excluded. The radio data provide relatively robust constraints on emission at early times throughout the explosion window, and only peak up in late 1996. The radio emission is fit with a modified ejecta-CSM interaction model (dashed lines, see $\S 4.3$ ), which deviates from the standard model in two important ways. First, the unusual sharp rise and variable spectral index cannot be fit by free-free absorption alone, and requires a $\gtrsim 100$ fold increase in radio luminosity as well. Second, the late-time slope remains flat up to the current epoch, rather than exhibiting the characteristic rollover seen in most RSNe. The late-time X-ray data show a relatively strong and significant rise between 2000-2007 both in the $0.5-2 \mathrm{keV}$ and 2-8 keV bands. This is very atypical of X-ray detected SNe and suggests that the blast wave of SN $1996 \mathrm{cr}$ is encountering a dense circumstellar shell. The dashed lines show empirical power-law fits to the data with labeled slopes. The early-time upper limits in all bands suggest that either very strong absorption played a role early on or more likely the progenitor of SN 1996cr formed a cavity during the final part of its evolution. The thin solid curves, the colors of which mirror those describing the SN 1996cr data, show the corresponding X-ray and radio light curves of SN 1987A for comparison (Hasinger et al. 1996; Manchester et al. 2002; Park et al. 2006). Only the luminosity and days after explosion axes apply to the SN 1987A data. The SN 1987A data have additionally been scaled up by a factor of 1,000 for illustrative purposes. While many of the specifics are different, the general trend of the SN 1996cr X-ray and radio light curves resembles those of SN 1987A. 


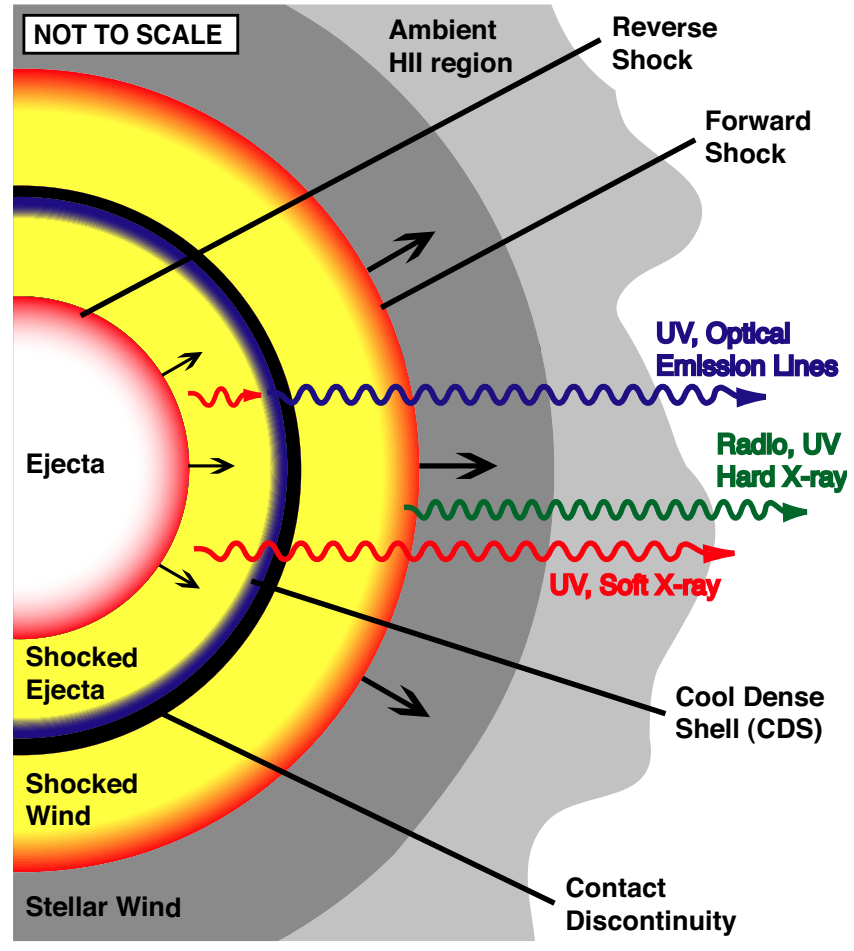

FIG. 7.- Idealized one-dimensional cartoon of a SN and its shocks, along with the stellar wind established CSM, and in the case of SN $1996 \mathrm{cr}$ a more distant $\mathrm{H}$ II region the $\mathrm{SN}$ is likely to be surrounded by. The forward shock and shocked CSM are thought to give rise to radio, UV, and hard X-ray emission $\left(T \sim 10^{9} \mathrm{~K}\right)$, while the reverse shock and shocked ejecta provide the bulk of the soft X-ray emission $\left(T \sim 10^{7} \mathrm{~K}\right)$. A CDS immediately behind the contact discontinuity can potentially absorb the soft X-ray emission, leading to a variety of photoionized emission lines. Not to scale.

sion which subsequently photoionizes a broad inner ejecta region from which many of the optical lines are believed to originate. When the CSM density is high and/or the power-law density distribution of the ejecta $\left(\propto R^{-n}\right)$ is steep, the reverse shock will remain radiative and a relatively thin, cool, dense, partially absorbing shell (CDS) should form between the reverse shock and the contact discontinuity. UV and X-ray radiation associated with the original shock breakout (detected for the first time from SN 2008D with Swift; Soderberg et al. 2008), as well as radiation from the ongoing forward and reverse shocks, may additionally photoionize the outer CSM. When the CSM is H-rich, this ionization should give rise to the strong, narrow-line $\mathrm{H}$ emission which is thought to epitomize the type IIn class (e.g., Schlegel 1990). To place SN 1996cr within the context of the above scenario, we examine our temporal and spectral data in detail.

\subsection{Optical Light Curve}

To assess the early history of SN 1996cr, we compared the $V$-band light curves for five type IIn, as well as 25 additional type II SNe of varying types, to the optical constraints detailed in $\S 2$ and plotted in Fig. 6. ${ }^{21}$ The three $V \gtrsim 15$ upper limits from R. Evans here provide some additional insight into the nature of SN 1996cr's light curve. We note that the light curves of type II SNe are relatively uniform and can generally

21 SNe light curves were acquired from http://virtual.sai.msu.su/ pavlyuk/snlcurve/. be divided into two distinct parts: (1) a sharp rise and variable decline rate between $\Delta V=0.005-0.035$ per day within the first $\sim 100$ days, followed by (2) a nearly universal decline from $M_{V} \sim-14$ with a rate of $\Delta V=0.003-0.01$ per day. The latter is thought to be linked to the decay of ${ }^{56} \mathrm{Co}$ (Turatto et al. 1990; Patat et al. 1994).

The above parameters define our average template light curve, and we use as our pivot point the constraints from 1996-03-16 to 1996-03-19, which lie 0-380 days from maximum. As a consistency check, we note that the slope between our 1996 and 1999 data points falls roughly within the expected range for type II SNe; we adopt $\Delta V=0.0035$ per day to fit our points. We find that R. Evans' constraint on 199503-31 excludes earlier explosion maximum dates for all but the faintest observed light curves, while the limit on 199508-13 excludes brighter than average observed light curves up to $\sim 50$ days prior to this date, and the limit on 1996-0303 fails to exclude any additional time. The dark grey region in the upper panel of Fig. 6 shows the range of parameter space which is still viable, demonstrating that these upper limits exclude SN 1996cr from being an extremely luminous type II SN over $\sim 15 \%$ of the explosion window. From this we argue that SN 1996cr ultimately had an uncorrected absolute $V$-band magnitude somewhere between -10.2 and -15.5. Once corrected for $\gtrsim 4.5$ magnitudes of extinction, SN $1996 \mathrm{cr}$ falls within the typical range for luminous type II SNe. At late times, SN 1996cr lies well above an extrapolation of the average decay rate, and is in fact $\sim 1.5$ magnitudes brighter in 2006 than in 1999. This fact demonstrates robustly that SN 1996cr must be buoyed either by circumstellar interaction or unresolved light echoes (e.g., Patat 2005; Patat et al. 2006). We discuss these two possibilities further in $\S 4.2$.

Several SNe (particularly of the IIn sub-type) have been shown to be strong near-IR emitters, due to reprocessed emission from hot dust (e.g., Gerardy et al. 2002; Pozzo et al. 2004). Whether the hot dust arises from preexisting dust in the circumstellar gas or from newly formed dust in the ejecta is still unclear. The HST NIC3 narrow-band constraints we have demonstrate that any near-IR emission above the earlier ESO near-IR imaging is likely to be minimal. Thus if a significant hot-dust component formed, it must have either been short-lived (e.g., destroyed by the initial UV flash from the SN or rapidly cooled) or occurred after the NIC3 observations in 1998. Mid-IR observations will ultimately constrain the dust-content of SN $1996 \mathrm{cr}$ better, since the bulk of anticipated dust is likely to be at temperatures of $\sim 100 \mathrm{~K}$ (the dust temperature in SN 1987A, for instance, is $\approx 180 \mathrm{~K}$; Dwek et al. 2008). While the pre-SN optical imaging does not provide useful constraints on the progenitor due to the strong extinction toward the Circinus Galaxy, the ESO near-infrared data does not suffer as much. The $J$-band limits in 1994 June are strong enough to exclude stars with $M_{\mathrm{J}} \lesssim-7.5$, effectively excluding the bright end of the luminous blue variable (LBV) distribution.

\subsection{Optical Spectrum}

As Fig. 1 demonstrates, the optical spectrum of SN 1996cr exhibits strong narrow emission lines of $\mathrm{H}$ and $\mathrm{O}$ that typify the type IIn SNe class (e.g., Schlegel 1990) superimposed on very broad emission complexes. The strong narrow lines suggest that circumstellar interaction plays an important role in the overall emission almost from the onset and, because of the strength of this emission, it can often mask the true nature of the photospheric emission. For instance, SN 1998 S (Fas- 


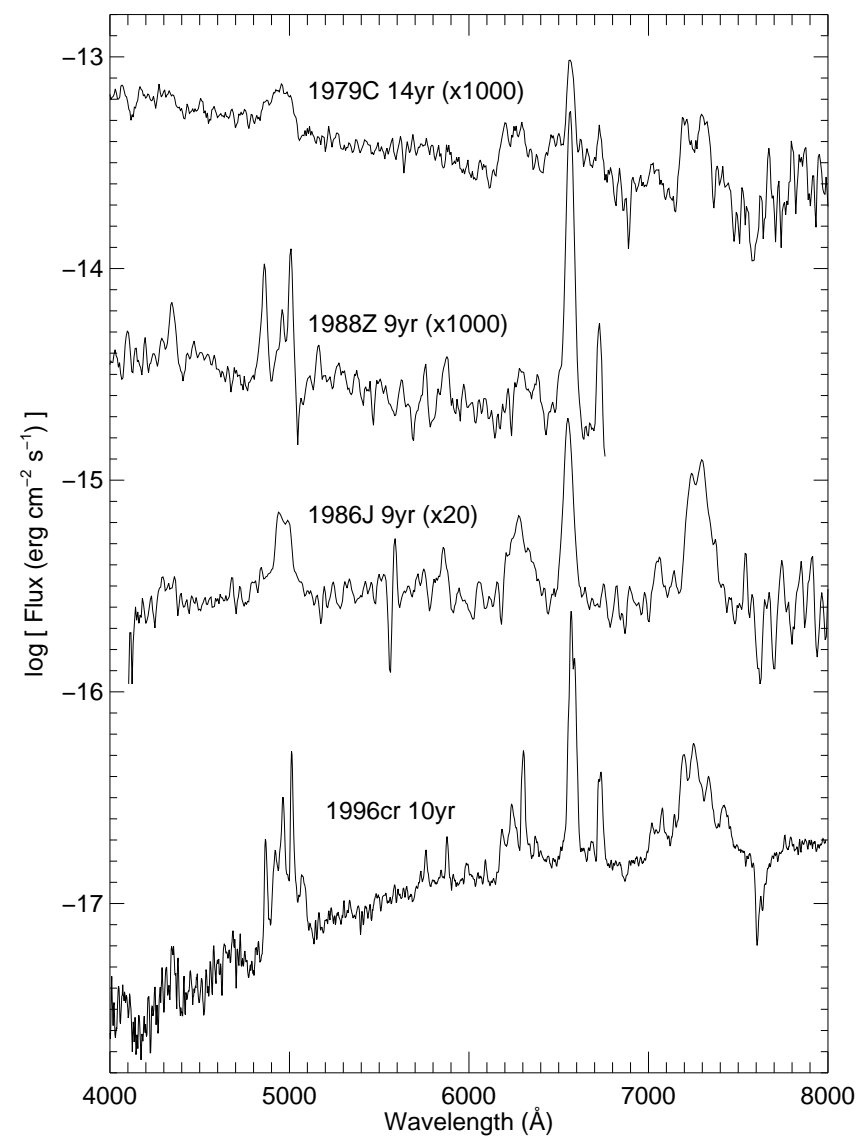

FIG. 8.- A comparison of the optical spectrum of SN 1996cr with those for a few other type IIn supernovae: SN 1979C (Leibundgut et al. 1991), SN 1986J (Fesen et al. 1999), SN 1988Z (Aretxaga et al. 1999). Spectra have been smoothed over a 3 pixel scale to facilitate easier comparisons. SN 1996cr has strong, narrow components similar to SN 1988 Z as well as broad asymmetric O complexes like SN 1979C and SN 1986J.

sia et al. 2001) was spectrally a type IIn and photometrically a type IIL, SN 2002ic (Hamuy et al. 2003; Deng et al. 2004) and SN 2005gj (Aldering et al. 2006; Prieto et al. 2008) were spectral "hybrids" showing type IIn-like H lines superposed on otherwise type Ia-like spectra, and SN 2001em (Chugai \& Chevalier 2006) spectrally evolved from a type Ic to a type IIn on the timescale of a few years.

In the case of SN $1996 \mathrm{cr}$, where we have limited temporal coverage during the early phases, we cannot deduce its early photometric or spectral type. We must rely solely on the latetime SN emission lines in our VLT spectrum to provide diagnostics on ejecta abundances and shock emission processes, as well as potential information on the mass-loss history and evolutionary status of the SN progenitor (e.g., CF94, Fransson et al. 2002, Chevalier 2005). As Fig. 8 shows, aside from the heavy extinction, the spectrum of SN 1996cr bears many striking similarities to the classic type IIn SNe, SN 1979C (Fesen et al. 1999), SN 1986J (Leibundgut et al. 1991), and SN $1988 Z$ (Aretxaga et al. 1999), and thus can probably be considered to be representative of the class as a whole.

We used the contributed IRAF package SPECFIT (Kriss 1994) to model the various spectral components of SN 1996cr. We began by fitting a power-law continuum, absorbed using the Galactic extinction curve of Cardelli et al. (1989) with
$R_{\mathrm{V}}$ fixed at $3.1 .^{22}$ However, since there is some degeneracy between the power-law slope and degree of extinction, we fixed $E(B-V)$ to a value of 1.8 determined by the narrowline Balmer decrement ${ }^{23}$ and then determined a best-fitted power-law slope of 3.4. This single powerlaw, however, underestimates the curvature of the continuum between 5500 $7000 \AA$ by $\approx 10 \%$, and thus conversely leads to overestimates in some line fluxes and widths. To fit the continuum more accurately, we instead adopted a broken power-law model with slopes of 2.65 above and 4.47 below a break at $6400 \AA$, respectively, yielding a statistically reliable model of the continuum over the entire spectrum. Note that the narrow Balmer lines are thought to arise from the unshocked progenitor wind and thus can be considered as the outer layer of emission. We therefore consider the extinction derived from the Balmer decrement to be a lower limit and caution that some interior components could suffer considerably higher extinction. We do not, however, see strong evidence of P Cygni absorption in any of the emission lines, suggesting that further absorption in the vicinity of SN $1996 \mathrm{cr}$ is probably minimal. This may also imply that the bulk of the CSM is fully ionized. We find only marginal evidence for $\mathrm{Na} \mathrm{D}$ absorption $(\sim 1.5 \sigma)$, which can be used to approximate the overall extinction (e.g., Turatto et al. 2003). The strength of this absorption appears too small compared to our other estimates, although the spectral resolution, signal-to-noise, and potential for contamination from complex line emission (see below) in the VLT spectrum severely compromise any meaningful constraint. We contend that the lack of broad-band blue and near-UV detections, compared to the strong blue continua typically seen in comparable SNe (e.g., SN 1979C, SN 1980K, SN 1988Z; Fesen et al. 1999; Immler \& Kuntz 2005), is fully consistent with our estimated extinction parameters and hence is reasonable.

We proceeded to fit all of the obvious emission lines with narrow Gaussian components. The parameters of each line were originally fit separately, but eventually the central wavelengths and FWHMs of the $\mathrm{H}, \mathrm{He}$, and heavier element lines were tied together as denoted in Table 7 to reduce free parameters and improve error estimates; this was justified by the fact that the free-parameter values overlapped with the nominal fixed ratio values to within errors. We note that there were, however, several large broad residual wings around the $\mathrm{H} \alpha$ and $\mathrm{O}$ lines. Thus we fit blended narrow and broad Gaussians, again linking central wavelengths and FWHMs as denoted in Table 7. We describe each element separately below.

We should caution that the late-time spectrum we observe for SN 1996cr could potentially be contaminated by earlytime SN spectral features due to the presence of unresolved light echoes from dust in the immediate circumstellar environment or in the intervening interstellar medium (e.g., Patat 2005; Patat et al. 2006). Notably, the spectra of SNe near maximum light are the most likely to continue on as light echoes, and thus we would expect such contamination to be isolated primarily to the continuum of SN $1996 \mathrm{cr}$ since earlytime spectra of SNe routinely exhibit either blue continua or psuedo-continua comprised of extremely broad emission lines which trace the high velocity ejecta (Filippenko 1997). For

\footnotetext{
22 While there is known to be some variance in the extinction curves from galaxy to galaxy (e.g., Calzetti et al. 1994), such differences should be relatively minimal above $4000 \AA$. Thus for simplicity we model the extinction using this particular Galactic extinction model since a significant fraction of the extinction comes from our own Galaxy.

23 Note that the decrement has a slight dependence on temperature and virtually none on density.
} 
TABLE 7

LINE IDENTIFICATIONS

\begin{tabular}{|c|c|c|c|c|c|c|}
\hline$\#$ & Line & Component & $F$ & $F^{c}$ & $\lambda_{c}$ & FWHM \\
\hline 1 & [O II] $\lambda 7319$ & $\mathrm{n}$ & $1.4 \pm 0.5$ & $49.6 \pm 17.2$ & $7324.9 \pm 1.4$ & $=29$ \\
\hline 2 & & $\mathrm{~b}$ & $13.6 \pm 1.0$ & $496.0 \pm 34.8$ & $7324.9(=1 *)$ & $=29$ \\
\hline 3 & & $\mathrm{c} 1$ & $6.2 \pm 0.5$ & $208.0 \pm 16.4$ & $7420.1\left(=30^{*}\right)$ & $=30$ \\
\hline 4 & & $\mathrm{c} 2$ & $19.3 \pm 0.7$ & $746.0 \pm 26.8$ & $7251.1(=31 *)$ & $=30$ \\
\hline 5 & & $\mathrm{c} 3$ & $16.8 \pm 0.5$ & $681.8 \pm 20.7$ & $7190.5(=32 *)$ & $=30$ \\
\hline 6 & [Ar III] $\lambda 7136$ & $\mathrm{n}$ & $1.8 \pm 0.2$ & $76.4 \pm 9.7$ & 7136.8 (f) & $=8$ \\
\hline 7 & He I $\lambda 7065$ & $\mathrm{n}$ & $3.1 \pm 0.3$ & $138.3 \pm 12.7$ & $7065.7(=21 *)$ & $=21$ \\
\hline 8 & {$[\mathrm{~S}$ II $] \lambda 6731$} & $\mathrm{n}$ & $3.6 \pm 0.3$ & $210.1 \pm 18.9$ & $6732.6 \pm 0.8$ & $741 \pm 24$ \\
\hline 9 & [S II] $\lambda 6716$ & $\mathrm{n}$ & $4.0 \pm 0.1$ & $239.0 \pm 7.8$ & $6717.6\left(=8^{*}\right)$ & $=8$ \\
\hline 10 & He I $\lambda 6678$ & $\mathrm{n}$ & $1.1 \pm 0.2$ & $70.0 \pm 13.8$ & $6675.7\left(=21^{*}\right)$ & $=21$ \\
\hline 11 & {$[\mathrm{~N} \mathrm{II}] \lambda 6583$} & $\mathrm{n}$ & $19.9 \pm 0.7$ & $1310.0 \pm 45.4$ & $6582.8 \pm 0.2$ & $=8$ \\
\hline 12 & $\mathrm{H} \alpha$ & $\mathrm{n}$ & $34.5 \pm 0.8$ & $2306.8 \pm 51.8$ & $6563.0 \pm 0.2$ & $669 \pm 18$ \\
\hline 13 & & $\mathrm{~b}$ & $9.5 \pm 1.0$ & $634.4 \pm 65.2$ & $6563.0(=12)$ & $4077 \pm 398$ \\
\hline 14 & [N II] $\lambda 6548$ & $\mathrm{n}$ & $6.4 \pm 0.0$ & $432.3 \pm 0.0$ & $6547.8(=11 *)$ & $=8$ \\
\hline 15 & {$[\mathrm{O} \mathrm{I}] \lambda 6300$} & $\mathrm{n}$ & $5.9 \pm 0.4$ & $486.7 \pm 30.7$ & $6296.2 \pm 0.4$ & $=8$ \\
\hline 16 & & $\mathrm{~b}$ & $5.3 \pm 0.6$ & $434.9 \pm 51.3$ & $6296.2(=15)$ & $=29$ \\
\hline 17 & & $\mathrm{c} 1$ & $2.1 \pm 0.3$ & $159.8 \pm 26.0$ & $6376.8\left(=30^{*}\right)$ & $=30$ \\
\hline 18 & & $\mathrm{c} 2$ & $5.8 \pm 0.4$ & $504.3 \pm 35.5$ & $6231.6(=31 *)$ & $=30$ \\
\hline 19 & & $\mathrm{c} 3$ & $2.9 \pm 0.3$ & $263.7 \pm 29.2$ & $6179.6(=32 *)$ & $=30$ \\
\hline 20 & {$[\mathrm{Fe} \mathrm{VII]} \lambda 6087$} & $\mathrm{n}$ & $0.5 \pm 0.2$ & $43.8 \pm 16.3$ & 6087.0 (f) & $=8$ \\
\hline 21 & He I $\lambda 5876$ & $\mathrm{n}$ & $1.6 \pm 0.2$ & $187.8 \pm 24.1$ & $5873.9 \pm 0.8$ & $913 \pm 65$ \\
\hline 22 & {$[\mathrm{~N} \mathrm{II}] \lambda 5755$} & $\mathrm{n}$ & $1.2 \pm 0.2$ & $152.3 \pm 22.2$ & $5755.0(\mathrm{f})$ & $=8$ \\
\hline 23 & {$[\mathrm{O} \mathrm{I}] \lambda 5577$} & $\mathrm{n}$ & $0.2 \pm 0.1$ & $35.6 \pm 15.4$ & $5579.0\left(=28^{*}\right)$ & $=8$ \\
\hline 24 & & $\mathrm{~b}$ & $0.3 \pm 0.2$ & $49.3 \pm 28.5$ & $5579.0\left(=28^{*}\right)$ & $=29$ \\
\hline 25 & & $\mathrm{c} 1$ & $0.3 \pm 0.2$ & $48.5 \pm 26.1$ & $5644.5\left(=30^{*}\right)$ & $=30$ \\
\hline 26 & & $\mathrm{c} 2$ & $0.3 \pm 0.1$ & $49.3 \pm 20.8$ & $5516.0(=31 *)$ & $=30$ \\
\hline 27 & & $\mathrm{c} 3$ & $0.3 \pm 0.2$ & $48.2 \pm 31.0$ & $5469.9(=32 *)$ & $=30$ \\
\hline 28 & [O III] $\lambda 5007$ & $\mathrm{n}$ & $5.2 \pm 0.3$ & $1638.6 \pm 92.3$ & $5007.9 \pm 0.3$ & $=8$ \\
\hline 29 & & $\mathrm{~b}$ & $3.5 \pm 0.4$ & $1099.1 \pm 130.3$ & $5007.9(=28)$ & $2795 \pm 174$ \\
\hline 30 & & $\mathrm{c} 1$ & $2.4 \pm 0.3$ & $682.5 \pm 71.4$ & $5066.4 \pm 1.1$ & $2049 \pm 19$ \\
\hline 31 & & $\mathrm{c} 2$ & $4.5 \pm 0.3$ & $1543.0 \pm 117.1$ & $4952.6 \pm 0.6$ & $=30$ \\
\hline 32 & & $\mathrm{c} 3$ & $1.9 \pm 0.3$ & $690.5 \pm 94.2$ & $4910.8 \pm 0.6$ & $=30$ \\
\hline 33 & [O III] $\lambda 4959$ & $\mathrm{n}$ & $1.6(=27 *)$ & $540.8(=27 *)$ & $4960.0\left(=28^{*}\right)$ & $=28$ \\
\hline 34 & & $\mathrm{~b}$ & $1.1\left(=28^{*}\right)$ & $362.7(=28 *)$ & $4960.0\left(=28^{*}\right)$ & $=29$ \\
\hline 35 & & $\mathrm{c} 1$ & $0.7(=29 *)$ & $225.2(=29 *)$ & $5018.9 !(=30 *)$ & $=30$ \\
\hline 36 & & $\mathrm{c} 2$ & $1.1\left(=30^{*}\right)$ & $509.2\left(=30^{*}\right)$ & $4905.2(=31 *)$ & $=30$ \\
\hline 37 & & $\mathrm{c} 3$ & $0.6\left(=31^{*}\right)$ & $227.9(=31 *)$ & $4866.8(=32 *)$ & $=30$ \\
\hline 38 & $\mathrm{H} \beta$ & $\mathrm{n}$ & $1.9\left(=11^{*}\right)$ & $745.0(=11 *)$ & $4861.1(=12 *)$ & $=12$ \\
\hline 39 & He II $\lambda 4685$ & $\mathrm{n}$ & $0.4 \pm 0.1$ & $221.8 \pm 59.6$ & $4684.5(=21 *)$ & $=20$ \\
\hline 40 & $\mathrm{H} \gamma$ & $\mathrm{n}$ & $0.4\left(=11^{*}\right)$ & $350.2\left(=11^{*}\right)$ & $4340.0(=12 *)$ & $=12$ \\
\hline
\end{tabular}

Note. - Column 1: Component number. Column 2: Emission line. Column 3: Component: Narrow (n), Broad (b), Complex (c\#). Column 4: Observed flux in units of $10^{-16} \mathrm{ergs} \mathrm{s}^{-1} \mathrm{~cm}^{-2}$. “*” indicates the flux of the line is tied to another line according to the relative ratio of their respective atomic line intensities. Column 5: Extinction-corrected flux in units of $10^{-16} \mathrm{ergs} \mathrm{s}^{-1} \mathrm{~cm}^{-2}$. "*" indicates the flux of the line is tied to another line according to the relative ratio of their respective atomic line intensities. Column 6: Central wavelength in $\AA$. “*” indicates the central wavelength of the line is tied to another line according to the ratio of their respective atomic rest wavelengths. Column 7: Gaussian Full-Width Half-Maximum in $\mathrm{km} \mathrm{s}^{-1}$.

TABLE 8

LINE RATIOS

\begin{tabular}{|c|c|c|c|c|c|c|}
\hline & Line Ratio & "n" & "b" & "c1" & "c2" & "c3" \\
\hline [O III] & $I(4959+5007) / I(4363)$ & $>5.40$ & $>3.62$ & $>5.08$ & $>2.25$ & $>2.28$ \\
\hline$[\mathrm{O} \mathrm{II}]$ & $I(3726+3729) / I(7319+7331)$ & $<703$ & $<3.02$ & $<1.78$ & $<7.46$ & $<1.90$ \\
\hline $\mathrm{O} \mathrm{I} /[\mathrm{O} \mathrm{II}]$ & $I(7774) / I(7319+7331)$ & $<0.50$ & $<0.16$ & $<0.12$ & $<0.48$ & $<0.34$ \\
\hline$[\mathrm{O} \mathrm{I}]$ & $I(6300+6364) / I(5577)$ & $>3.95$ & $>1.90$ & $>3.14$ & $>1.07$ & $>1.63$ \\
\hline [S II] & $I(6731) / I(6716)$ & $0.88_{-015}^{+0.18}$ & & & & \\
\hline [S II] & $I(6716+6731) / I(4068+4076)$ & $>0.31$ & & & & \\
\hline$[\mathrm{N} \mathrm{II}]$ & $I(6548+6583) / I(5755)$ & $11.44_{-3.75}^{+9.54}$ & & & & \\
\hline [Ar III] & $I(7136+7751) / I(5192)$ & $>0.84$ & & & & \\
\hline He I & $I(7065) / I(5876)$ & $0.74_{-0.35}^{+0.78}$ & & & & \\
\hline He I & $I(7065) / I(6678)$ & $1.98_{-1.07}^{+4.15}$ & & & & \\
\hline $\mathrm{He} \mathrm{I}$ & $I(6678) / I(5876)$ & $0.37_{-0.26}^{+0.59}$ & & & & \\
\hline
\end{tabular}

Note. - Column 1: Line-intensity ratio. Columns 2-6: Fitted component values. 


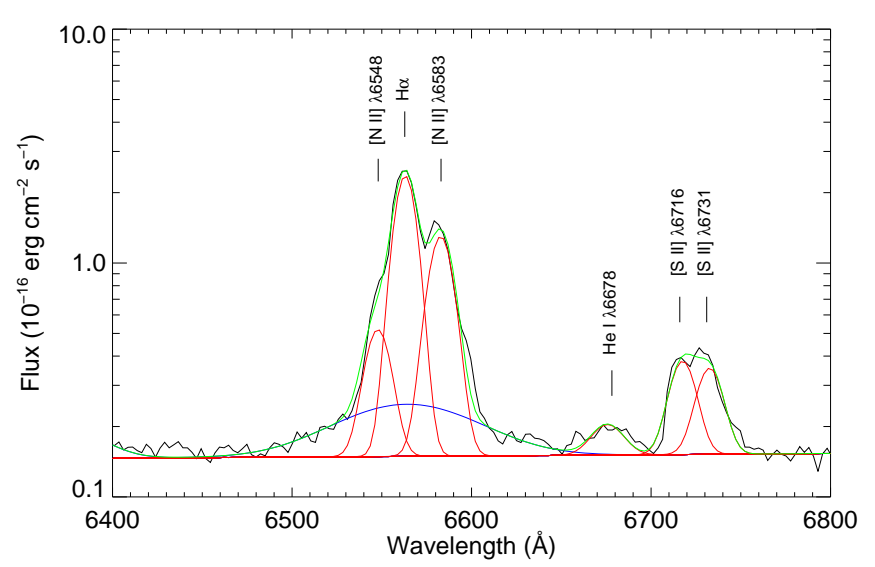

FIG. 9.- A section of the spectrum in Fig. 1 showing in detail the fitted components in the vicinity of the $\mathrm{H} \alpha$ line. Obvious emission lines have been identified and an empirical model has been fit to the data as described in $\S 4.2$; again the green line shows the overall fit to the spectrum while the red (blue) lines show the individual narrow (broad) components. Although the narrow $\mathrm{H} \alpha$ strongly overlaps with the $\mathrm{N}$ II doublet, our spectral resolution is sufficient to deblend it.

SN $1996 \mathrm{cr}$, we find only distinct emission lines of low-tomoderate velocity (i.e., $<5,000 \mathrm{~km} \mathrm{~s}^{-1}$ ) on top of a smooth continuum, with the only notable exception being the region immediately surrounding He $\lambda 5876$. The equivalent widths of these emission lines are also comparable to other well-studied SNe such as those in Fig. 8, suggesting at least that SN 1996cr is not unusual. We additionally included a few early-time light curves of typical $\mathrm{SNe}$ in our spectral fitting, and found that aside from some degeneracy with our continuum model, such spectra are not generally compatible. Thus we argue that the majority of our line estimates, with the possible exception of $\mathrm{H} \alpha$, are unlikely to be strongly contaminated. In the case of $\mathrm{H} \alpha$, there are rare exceptions, such as the type IIn SN 1994W and SN 1994Y, where early spectra display bright, relatively narrow $\mathrm{H}$ lines on top of blue continua from the outset (e.g., Wang et al. 1996; Chugai et al. 2004). In these cases, the narrow lines are interpreted as arising from the SN shock transmitted into a dense, clumpy CSM very close in to the $\mathrm{SN}\left(\sim 10^{15} \mathrm{~cm}\right)$. Our radio and X-ray constraints at early times suggest this is an unlikely scenario for SN $1996 \mathrm{cr}$ (see $\S 4.3$ and $\S 4.4)$. However, since we lack early optical spectra of SN 1996cr, interpretation of the $\mathrm{H} \alpha$ emission should be viewed with some discretion.

\subsubsection{Hydrogen}

We find SN 1996cr exhibits both narrow $\left(669 \pm 18 \mathrm{~km} \mathrm{~s}^{-1}\right)$ and broad $\left(4077 \pm 398 \mathrm{~km} \mathrm{~s}^{-1}\right)$ components of $\mathrm{H} \alpha$, with fluxes of $(2.31 \pm 0.05) \times 10^{-13} \mathrm{ergs} \mathrm{s}^{-1} \mathrm{~cm}^{-2}$ and $(6.34 \pm 0.65) \times 10^{-14} \mathrm{ergs} \mathrm{s}^{-1} \mathrm{~cm}^{-2}$, respectively. This portion of the spectrum is shown in detail in Fig. 9. The narrow line appears to be marginally resolved above our estimated instrumental resolution, although this fact should be regarded with some caution since the line is strongly blended with $\mathrm{N}$ II; we await confirmation via high-resolution spectroscopic followup.

The flux found in our VLT spectrum is consistent to within errors with that measured from the $H S T$ F656N filter seven years earlier, indicating that the overlapping $\mathrm{H}$ II region still only contributes at most $30 \%$ to the total narrowline flux above and $10 \%$ to the broad-line flux. Accounting for this contamination, we find corresponding extinction- corrected luminosities of $(2.79 \pm 0.06) \times 10^{38} \mathrm{ergs}^{-1}$ and $(9.86 \pm 0.10) \times 10^{37} \mathrm{ergs} \mathrm{s}^{-1}$ for the narrow and broad components, respectively. The energy generated in either the narrow or broad $\mathrm{H} \alpha$ lines is significantly above that predicted by the decay of ${ }^{56} \mathrm{Co}-{ }^{56} \mathrm{Fe}$ at this late stage (e.g., Chugai 1991), and therefore must instead be related either to mechanical energy associated with the $\mathrm{SN}$ ejecta-wind interaction or potential light echoes.

The line width and systemic velocity of the narrow component argue for an origin beyond the blast wave, associated with either the shocked or unperturbed progenitor wind (e.g., Fransson et al. 2002). If the line does arise from a dense, preSN, H-rich CSM, it must have been photoionized and heated by UV and X-ray radiation from the massive progenitor and the initial shock breakout of the SN. The fact that the $\mathrm{H} \alpha$ luminosity has remained relatively constant between the epochs of the VLT and HST observations suggests that this region is now being sustained by ionizing flux from the current ejectawind shock zone (see $\S 4.4$ ), although again contaminating echoes from prior epochs is a concern here. For comparison, the $\mathrm{H} \alpha$ flux of other type IIn SNe such as SN 1988Z, SN 1995G, and SN 1995N decreased by at least an order of magnitude in $\sim 4$ yrs (Aretxaga et al. 1999; Pastorello et al. 2002; Fransson et al. 2002). The $\mathrm{H} \alpha$ luminosity thus provides an important diagnostic on the total ionizing radiation that complements the X-ray observations, as this line arises as a result of recombination and collisional excitation. If this $\mathrm{H} \alpha$ component is associated with CSM interaction and is powered by photoionization, then the X-ray luminosity from the forward and reverse shocks should roughly be proportional to the $\mathrm{H} \alpha$ luminosity following Eq. 3.7 in CF94 such that

$$
L(\mathrm{H} \alpha) \approx 4.4 \times 10^{37}\left(T / 10^{7} \mathrm{~K}\right)^{-1}\left(L_{\mathrm{X}} / 10^{40}\right) \mathrm{ergs} \mathrm{s}^{-1} .
$$

This implies $L_{X}=7.0 \times 10^{40}\left(T / 10^{7} K\right)$ ergs $\mathrm{s}^{-1}$, however, which is two orders of magnitude larger than the product of observed absorption-corrected X-ray luminosity and temperature. This would argue for the soft X-rays from the reverse shock, which should dominate bolometrically, to perhaps be radiative and hence reprocessed by either unshocked lowdensity ejecta or high-density material in the CDS (this possibility is discussed further in \$4.2.2). Alternatively, the narrow line could arise from shocks being driven into dense, slowmoving clumps associated with the progenitor wind. This would more naturally explain the discrepancy in observed and estimated ionizing flux, in which case the line is instead powered by collisional excitation.

If the narrow $\mathrm{H} \alpha$ stems from the stellar wind, then the $\mathrm{H} \alpha$ luminosity can also be related to the mass of the unshocked wind as

$$
L(\mathrm{H} \alpha)=(1-5) \times 10^{39}\left(\dot{M}_{-4} / v_{w, 1}\right)^{2} r_{18}^{-1} \operatorname{ergs~s}^{-1},
$$

where $r_{18}$ is the radius in units of $10^{18} \mathrm{~cm}, v_{w}$ is the wind velocity in units of $10 \mathrm{~km} \mathrm{~s}^{-1}$, and $\dot{M}_{-4}$ is the mass-loss rate in units of $10^{-4} \mathrm{M}_{\odot} \mathrm{yr}^{-1}$ (Deng et al. 2004; Wang et al. 2004). We assume an emissivity based on Case $\mathrm{B}$ recombination at a temperature of $10,000 \mathrm{~K}$, which is in rough agreement with our extinction-corrected $\mathrm{H} \alpha / \mathrm{H} \beta / \mathrm{H} \gamma$ ratios. Our $\mathrm{H} \alpha$ luminosity implies a mass-loss rate of $\sim 10^{-5} v_{w, 1} r_{18}^{0.5} \mathrm{M}_{\odot} \mathrm{yr}^{-1}$. For a typical red supergiant lifetime of $\gtrsim 10^{5} \mathrm{yr}$, this implies that at least a few $\mathrm{M}_{\odot}$ of hydrogen-rich matter was cast off into the CSM. 
The high-velocity component of $\mathrm{H} \alpha$, on the other hand, must be related directly to the blast wave in order to have achieved its present dispersion. This component could be associated with the reverse shock or CDS behind the reverse shock, perhaps from swept-up wind material or hydrogen that remained on the surface of the progenitor. The broad-line $\mathrm{H} \alpha$ velocity is thought to provide a lower limit to the true shock velocity. However, an observed disperion of $\sim 4,000 \mathrm{~km} \mathrm{~s}^{-1}$ is much lower than the $15,000-40,000 \mathrm{~km} \mathrm{~s}^{-1}$ initially expected for a typical core-collapse $\mathrm{SN}$, and implies that the shock in SN 1996cr has already slowed down substantially due to circumstellar interaction. This observed velocity decrease of at least $\gtrsim 4-10$ implies a circumstellar density increase of $\gtrsim 16$ 100. This line is almost certainly powered by collisional excitation associated with shocked ejecta.

We additionally see significant narrow-line emission in $\mathrm{H} \beta$ and $\mathrm{H} \gamma$, as well as marginally in $\mathrm{H} \delta$. Broad emission components could also exist for these lines; however, for typical line ratios with respect to $\mathrm{H} \alpha$ they would be marginal at best. Additionally, such emission would be impossible to deblend from the $[\mathrm{O} \mathrm{III}]$ emission complexes.

\subsubsection{Oxygen}

We find evidence for one narrow-line and four broad-line velocity components in the profiles of the [O III] $\lambda \lambda 5007,4959,[\mathrm{O}$ I] $\lambda 6300$, and [O II] $\lambda 7319$ lines as shown in Fig. 10, with velocities of $3577 \pm 65$ ("c1"), $66 \pm 18$ ("n", "b"), $-3247 \pm 36$ ("c2"), and $-5755 \pm 37 \mathrm{~km} \mathrm{~s}^{-1}$ ("c3"). We note there is also marginal evidence for similar multi-component emission complexes associated with the [O I] $\lambda 5577$ and [O III] $\lambda 4363$ lines. This is somewhat in contrast to the late-time spectra of many type II SNe such as SN 1993J, which show evidence for more irregular, "clumpy", newly synthesized O complexes (Matheson et al. 2000).

A few type II SNe have shown evidence for three-peaked profiles, which have either been ascribed to inhomogeneous, partially absorbed disk- or torus-shaped CSMs (e.g., Gerardy et al. 2000; Leonard et al. 2000), or from line formation in the CDS (e.g., Fransson et al. 2005). In such cases, however, the profiles arise not from the $\mathrm{O}$ lines, but from $\mathrm{H} \alpha$, $\mathrm{Mg}$ II, or Ly $\alpha$; this may indicate that SN 1996cr is O-rich. Nonetheless, the first scenario typically yields two relatively symmetric positive and negative velocity peaks indicative of emission from an equatorial ring, while a third central peak could emerge due to a population of dense clouds embedded in a much less dense CSM. In the second scenario, the CDS can only lead to such peaks under a very specific set of conditions such that it is very optically thick to such line emission, it is thin enough that the velocity gradient over the shell is small compared to the thermal velocity, and finally it has a macroscopic velocity larger than its thermal velocity. The CDS could be clumpy, lowering the filling factor and allowing some emission to escape. A final possibility for this velocity structure could arise from the SN blast wave impacting a dense shell produced by a wind-blown bubble. In this case, several secondary shocks could separate the CSM into multiple concentric shells (e.g., Dwarkadas 2005), such that each peak may represent a different velocity shell. The inner (redshifted) shells would be preferentially absorbed compared to the outer (blue-shifted) shells, just as we observe.

For SN 1996cr, we see a broad central peak ("b", FWHM $=2795 \pm 174 \mathrm{~km} \mathrm{~s}^{-1}$ ) and two roughly symmetric pos-

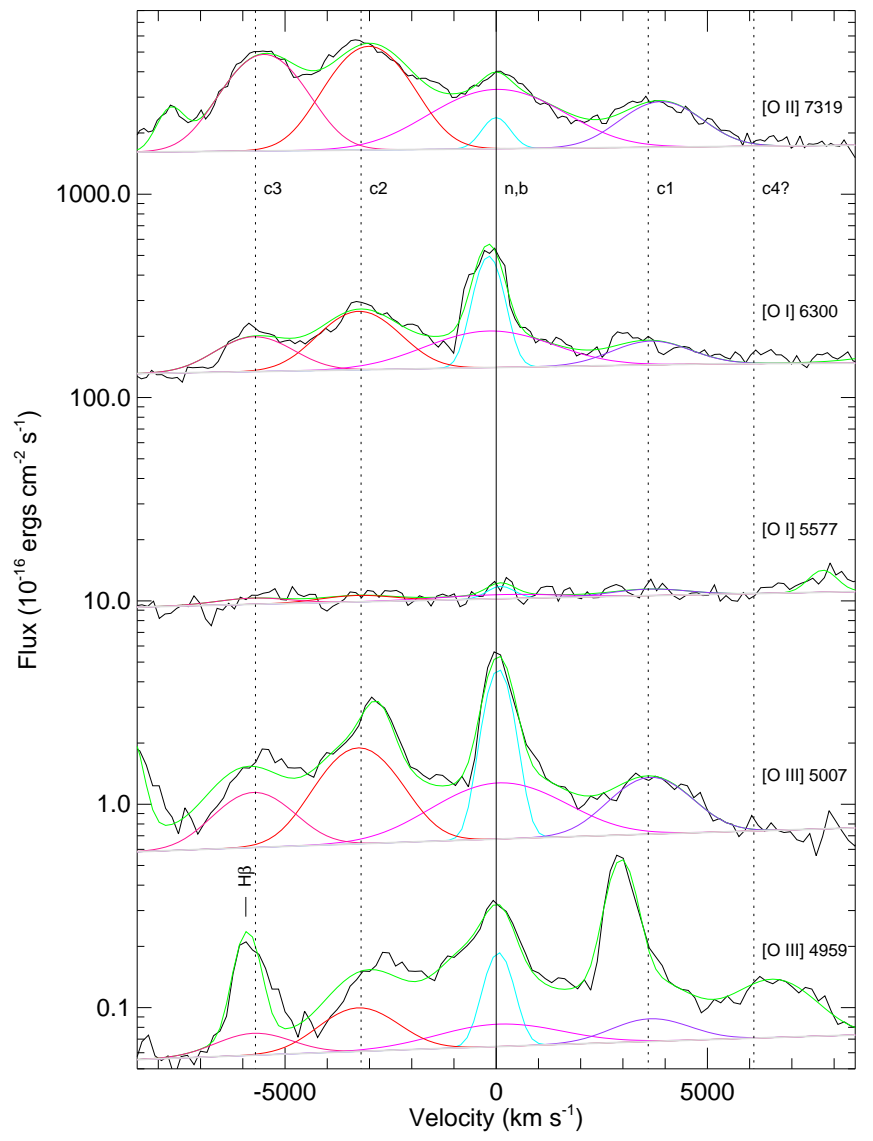

FIG. 10.- Velocity structure of $\mathrm{O}$ emission lines in units of $\mathrm{km} \mathrm{s}^{-1}$. The VLT spectra in the vicinity of five labeled complexes (black) and their respective model fits (green showing the overall full component fit, while contributions from a distinct velocity component are shown in different colors) are shown. Each spectrum is offset by a factor of 10 for clarity. The solid vertical line denotes the approximate position of the stationary narrow (cyan) and broad (magenta) components, while the dashed vertical lines denote the expansion velocities of components $\mathrm{c} 1$ (purple), c2 (red), and c3 (pink); a potential symmetric component $\mathrm{c} 4$ is not evident in the data. Although several of the lines are strongly blended, each of the components remains relatively distinct. The line components for $\lambda 5007$ and $\lambda 4959$ overlap such that in a few instances no single component obviously dominates.

itive and negative peaks ("c1" and "c2", FWHM= $2049 \pm$ $19 \mathrm{~km} \mathrm{~s}^{-1}$ ). The red peak is always fainter than the blue one, implying significant internal extinction. Interestingly, we also see a fourth, faster negative component ("c3") which does not have an obvious positive counterpart; with our current sensitivity such a symmetric component (i.e., "c4?") must be at least a factor of 2.5 fainter $(3 \sigma)$, implying significant additional internal absorption above that of the lower velocity peaks, if it exists. This "c3" component could be indicative of further asymmetry in the structure of SN 1996cr. We need only look to the nearby SN 1987A, with its triple ring structure, for an example of such potential complexity (McCray 1993, 2007). While the properties of the CSM of SN 1996cr appear to differ dramatically from those of SN 1987A, the spectral features hint at an environment that is equally dynamic and exotic. Another possibility is that the "c3" component represents a light echo from an earlier epoch, where the $\mathrm{O}$ lines were shifted by a larger velocity and more heavily absorbed. We reject this, however since it would require the "c3" oxygen features to peak strongly in the blue part of the spectrum, while we detect them clearly throughout the spec- 
trum.

Notably, when we apply our five-component model to the $\mathrm{O}$ lines, we find that our constraints on [O I] $\lambda 6363$ are consistent with zero flux if we fit [O I] $\lambda 6363$ and [O I] $\lambda 6300$ separately. Given the degeneracy between the potential broad components of these two lines though, this only provides weak $I(\lambda 6363) / I(\lambda 6300)$ constraints for the various components (n: $<1.0, \mathrm{~b}:<0.8, \mathrm{c} 1<0.6, \mathrm{c} 2<1.5, \mathrm{c} 3<0.9)$. Such ratios are all fully consistent with the normal transition probability of 0.3 . For the following, we fit only $\lambda 6300$ and use its flux to estimate the total intensity $I(\lambda 6300+\lambda 6363)$ for the various components. As such we may incur some small additional errors associated with flux contamination between different velocity components of the two lines. We also caution that the complex which we attribute solely to [O II] $\lambda 7331$ is likely in fact to be a blend of [O II] $\lambda \lambda \lambda 7319,7331$ and [Ca II] $\lambda \lambda 7291,7325$. Using our five-component model, however, we find that additional lines do not significantly improve $\chi^{2}$. The relative symmetry and constraints on line centers of the individual components imply that other lines do not contribute strongly to the overall emission, although notable small systematic residuals suggest minor blending is likely. The overlapping broad-line $\mathrm{O}$ profiles seen in Fig. 10 appear to be overestimating the valleys in between distinct component peaks, indicating that either the profiles are not represented well by Gaussians or that P Cygni-like absorption may be present but masked by the overall complexity of the emission. Higher-resolution spectroscopy is needed to place better constraints on these potential emission and absorption contributions.

The fact that the various components show up in all of the $\mathrm{O}$ lines strongly argues for a scenario in which they arise from physically associated regions within the SN. Since we see several ionization states though, it is likely that there is strong stratification within these distinct spatial regions (very likely due to an extended partially-ionized zone; e.g., CF94) and it is only appropriate to employ nebular-to-auroral line ratios for a given ionic species to estimate physical conditions. However, our constraints on $\lambda \lambda 3726,3729, \lambda 4363$, and $\lambda 5577$ are poor and do not provide physically interesting temperature and density limits. Table 8 lists these oxygen ratio constraints. Notably, the relative observed strengths of the $\mathrm{O}$ lines are broadly consistent with the model predictions of CF94 (see Figs. 4 and 5), whereby the modeled ejecta is photoionized by X-rays from the circumstellar interaction.

Finally, there appears to be no sign of the O I $\lambda 7774$ recombination line either at its systemic value or other velocities seen in the [O I] lines (although telluric absorption does hinder assessment of the blue components). This suggests that the narrow $\mathrm{O}$ resides in a physically distinct region separate from the narrow $\mathrm{H} \alpha$, as one typically sees a $10^{-3}$ ratio. The broad, high-velocity $\mathrm{O}$ emission is not mirrored in any other elements, although there is possibly some complexity to the He emission (see below), further arguing that it probably arises from an O-rich shell of processed gas associated with freely expanding ejecta.

\subsubsection{Helium}

SN 1996cr shows evidence for four prominent, narrow $\mathrm{He}$ lines $\left(906 \pm 101 \mathrm{~km} \mathrm{~s}^{-1}\right)$ : He I $\lambda 7065$, He I $\lambda 6678$, He I $\lambda 5876$, and He II $\lambda 4686$. The narrow-line width is marginally broader than that of $\mathrm{H} \alpha$, hinting that it may be spatially distinct. The He I line ratios do not strongly constrain the electron tem- perature and density of the gas. The emissivities of Porter et al. (2005) constrain the $\mathrm{He}$ gas to be at $T \gtrsim 10^{4} \mathrm{~K}$ and $n_{e} \gtrsim 10^{4} \mathrm{~cm}^{-3}$.

We see substantial broad residual emission around $\lambda 7065$ and $\lambda 5876$ which currently defies identification. Unlike the well-formed velocity components associated with the various $\mathrm{O}$ lines, there appears to be no spectral consistency between different He lines. For instance, the emission around $\lambda 7065$ appears box-like and quite strong relative to the systemic narrow line, while the emission around $\lambda 5876$ appears more spread out and much fainter relative to the systemic narrow line. Thus if this broad emission is associated with $\mathrm{He}$, it is likely present in several regions of the $\mathrm{SN}$, and may be far more complex than $\mathrm{H}$ or $\mathrm{O}$ are. There may be additional marginal broad features around $\lambda 4686$, while any potential features around $\lambda 6678$ are masked by the strong, broad $\mathrm{H} \alpha$ emission.

The narrow He emission likely arises from the same regions of the progenitor wind as the narrow hydrogen emission, whereas the broad unidentified emission, if it comes from helium, could be swept up material or expelled clumps. Either way, its complex profile suggests it is much more irregularly distributed compared to $\mathrm{H}$.

\subsubsection{Nitrogen}

We find evidence for strong, narrow $\mathrm{N}$ emission in the spectrum of SN 1996cr in the form of both the nebular [N II] $\lambda \lambda 6583,6548$ (which appear as a resolvable blend with $\mathrm{H} \alpha$ ) and the auroral [N II] $\lambda 5755$ lines. The lines have a best-fitted FWHM of $741 \pm 24 \mathrm{~km} \mathrm{~s}^{-1}$. The line-intensity ratio $I(\lambda 6583+\lambda 6548) / I(\lambda 5755)$ implies an electron density of $n_{e} \gtrsim 1 \times 10^{5} \times\left(10^{4} / T\right)^{0.5} \mathrm{~cm}^{-3}$ for $T \lesssim 20,000 \mathrm{~K}$ (Osterbrock 1989; Keenan et al. 2001). The high density suggests that clumping of $\mathrm{N}$ is likely. As argued for the narrow $\mathrm{H}$ emission, the majority of the emission must arise from the SN-CSM interaction given the pre-SN imaging constraints. The implied velocity of the lines suggest it is likely to have a circumstellar origin, while the strength of the emission hints at potential nitrogen enrichment, as has been notably seen in several other type II SNe such as SN 1979C, SN 1987A, SN 1993J, SN 1995N, and SN 1998S (Fransson et al. 1984, 2002, 2005).

\subsubsection{Sulfur}

The spectrum shows strong, narrow [S II] $\lambda \lambda 6731,6716$ emission lines with a best-fitted FWHM of $741 \pm 24 \mathrm{~km} \mathrm{~s}^{-1}$, which again clearly must come from the SN-CSM iteraction given the pre-SN imaging constraints. This feature is relatively uncommon in late-time type II SNe spectra, although it is seen in several comparatively-aged type IIn SNe spectra such as SN 1988Z and SN 1979C (see Fig. 8). The [S II] lineintensity ratio $I(\lambda 6731) / I(\lambda 6716)$ implies an electron density of $n_{e}=400_{-60}^{+100} \times\left(10^{4} / T\right)^{0.5} \mathrm{~cm}^{-3}$ (Osterbrock 1989). We additionally see a hint of the [S II] $\lambda \lambda 4076,4069$ doublet, although the significance of this feature is marginal and strongly affected by our estimated extinction value. Given the strong density contrasts, this component should reside in a different region from $[\mathrm{N}$ II $]$.

\subsubsection{Argon}

Narrow Ar emission is present in the spectrum of SN 1996cr in the form of both the nebular

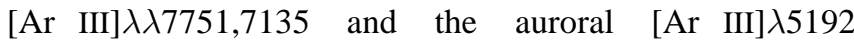
lines. Our lower limit to the line intensity ratio 
$I(\lambda 5192) / I(\lambda 7751+\lambda 7135)$, however, is too loose to place any useful constraint on the electron temperature or density of this component (e.g., Keenan et al. 1988).

\subsubsection{Iron}

We find significant high-ionization [Fe VII] $\lambda \lambda 6087,5721$ lines, but only a marginal [Fe VII] $\lambda 5158$ line. Additionally, [Fe X] 76375 may be present, although it is currently lost amid the broad [O I] emission. The lack of a strong $\lambda 5158$ line constraint unfortunately leads to physically uninteresting electron-density limits (e.g., Keenan \& Norrington 1987, 1991).

\subsubsection{Unidentified}

We also see tentative features at $\lambda 5840, \lambda 5980, \lambda 7011$, and $\lambda 7785$ which remain unidentified.

\subsection{Radio Light Curve}

An expectation from the interaction between the blast wave and the CSM is the production of copious non-thermal synchrotron emission from relativistic electrons and enhanced magnetic fields within the thin shell trailing the forward shock. The radio emission should be proportional to the injection spectrum of electrons and various loss mechanisms, which are effectively determined by the composition and density of the CSM. At early times, however, the radio emission can be absorbed via free-free (FF) or synchrotron selfabsorption (SSA) depending on the magnetic field strength and CSM density and structure. Then as the shock wave overtakes more and more of the CSM, the radio emission becomes progressively less absorbed, leading to a characteristic frequency-dependent "turn on" first at higher frequencies and later at lower ones.

Following the prescription of Chevalier (1982b) the optically thin radio luminosity of this shell can be described by

$$
L_{\nu} \propto 4 \pi R^{2} \Delta R K B^{(\gamma+1) / 2} \nu^{(\gamma-1) / 2} e^{-\tau}
$$

where $L_{\nu}$ is the radio luminosity at frequency $\nu, \Delta R$ is the thickness of the synchrotron emitting region at radius $R, B$ is the strength of the magnetic field, the distribution of accelerated particles is assumed to take a power-law form of $N(E)=K E^{-\gamma}$, and $\tau$ is the absorption opacity. Both $B$ and $K$ scale with the thermal pressure $P$, which itself scales with the CSM density $\rho_{\text {CSM }}$ of the synchrotron emitting region. The opacity $\tau$ likewise scales with $\rho_{\mathrm{CSM}}$, although in the case of FF absorption it is proportional to the integrated density along the line of sight. We assume here that all absorbing media are purely thermal, ionized hydrogen with opacities proportional to $\nu^{-2.1}$. We contend that the FF opacity dominates over the SSA opacity given that (1) the shock velocity required by SSA, as implied by the radio luminosity and time of peak emission (see, e.g., Chevalier et al. 2006, and references therein), is many times lower than either the optically inferred late-time shock velocity or the expected early-time shock velocity, and (2) the absorption does not deviate substantially from $\tau \propto \nu^{-2.1}$ where it is possible to constrain it, as expected for FF. By comparison, SSA should scale as $\tau_{\text {SSA }} \propto \nu^{\alpha-2.5}$, which is too steep to fit any of our well-sampled epochs. In this case, the FF opacity is given by

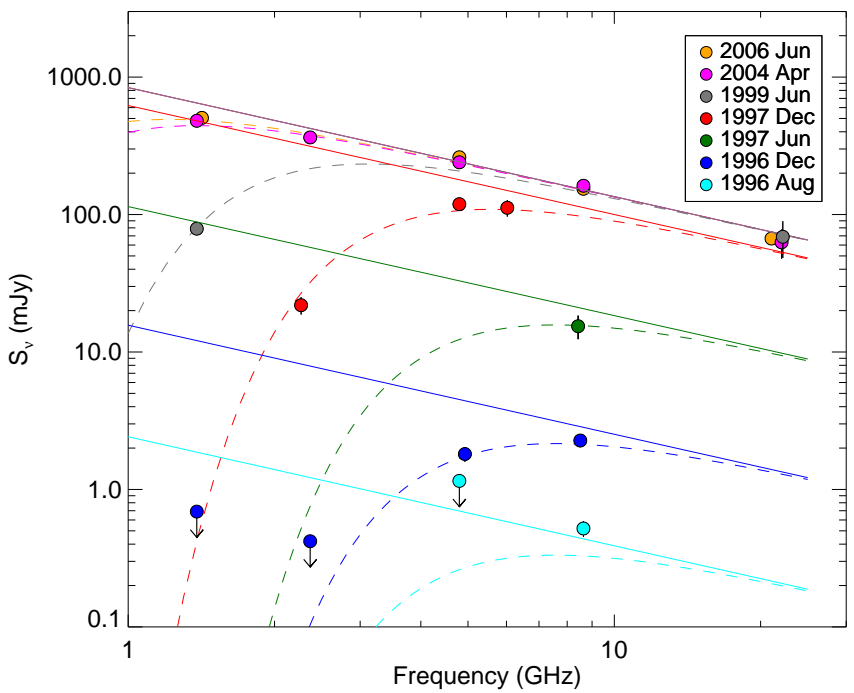

FIG. 11.- Radio spectra of SN 1996cr over several epochs. Solid circles denote data, while solid and dashed lines denote intrinsic and absorbed radio spectra from our best-fitting model. We find dramatic changes in the low-frequency slope indicative of time-varying absorption, but relatively little change to the high-frequency slope additionally suggesting a strong increase in the CSM density.

$$
\tau_{\mathrm{ff}} \approx 3.28 \times 10^{-7}\left(\frac{T_{e}}{10^{4} \mathrm{~K}}\right)^{-1.35}\left(\frac{\nu}{1 \mathrm{GHz}}\right)^{-2.1}\left(\frac{E M}{\mathrm{pc} \mathrm{cm}^{-6}}\right)
$$

where the emission measure, EM, is given as

$$
E M=\int_{r}^{\infty} N_{e}^{2} d r=\int_{r}^{\infty}\left(\frac{\rho_{\mathrm{CSM}}}{\mu_{\mathrm{H}} m_{\mathrm{H}}}\right)^{2} d r
$$

Thus the unabsorbed radio luminosity $L_{\nu}$ and FF opacity $\tau_{\text {ff }}$ afford us two completely independent opportunities to trace the CSM density profile.

With the above in mind, we now examine the radio data for SN 1996cr. Fig. 6 demonstrates that there are strong upper limits at four observable frequencies from the earliest time of explosion up through the end of 1996. These non-detections imply either strong early absorption or the presence of a lowdensity cavity. Over the next year, we see a dramatic rise in the radio emission, first at higher frequencies and later at lower ones. The inverted rise, however, is atypical and cannot solely be the result of dwindling absorption, as is common in other RSNe. To make this point clearer, we present the radio spectrum of SN 1996cr for several epochs in Fig. 11. Much of the radio data were taken separately, and thus a few of our adopted late-time epochs actually span a severalmonth window; such an approach is validated by the fact that strong, rapid radio variability is not expected or observed at late times.

From Fig. 11, we see that the data taken during the 2006 June and 2004 April epochs are only minimally absorbed and thus provide a solid constraint both on the intrinsic synchrotron spectral index $(\alpha=-0.79 \pm 0.02)$ and the FF absorption beyond the synchrotron-emitting region. Using our model radio spectra to guide the eye (described below), we see the presence of increasing absorption at the lowest frequencies as we progress back through the 1999, 1997, and 1996 epochs. This is a natural consequence of the radio-emitting 
region overtaking the CSM which absorbs it. Importantly, the observed spectral index between $4.8 \mathrm{GHz}$ and $8.5 \mathrm{GHz}$ does not change dramatically between 1996 and 2006. If we assume that the intrinsic spectral index is constant, this implies that the spectrum here is optically thin. The $8.5 \mathrm{GHz}$ emission is thus only modestly affected by FF absorption $(\sim 20 \%)$ even at early epochs and can be considered a relatively robust tracer of the intrinsic radio luminosity at all times. As such, the $8.5 \mathrm{GHz}$ flux density appears to jump by a factor of $\approx 150$ between 1996 August and 1997 December (515 days) and an additional factor of $\approx 1.33$ between 1997 December and 2004 April (2384 days). Given that the radio-emitting region is spread over relatively large scales, the radio emission should vary smoothly in time. Empirically, we find that a broken power law, with indices $a_{1}=8.76 \pm 0.24$ and $a_{2}=0.71 \pm 0.09$ between the three highly constrained epochs above, provides an adequate fit to the data from other epochs aside from 1996 August $\left(\chi_{\nu}^{2}=2.65\right)$, although there is clearly some degeneracy with other parameters such as $\alpha$ and $\tau_{\text {ff }}$ (see Fig. 11). This model should at least provide a qualitative understanding of the light curve during this overall period.

The early upper limits and dramatic increase in the radio luminosity imply a sharp rise in $\rho_{\mathrm{CSM}}$ as well, highlighting the possible transition from a fast, sparse stellar wind to a slow, dense one. Such transitions typically lead to the formation of a wind-blown bubble (e.g., Weaver et al. 1977; Garcia-Segura et al. 1996a,b), and numerous researchers have explored the subsequent interaction between the complex CSM associated with a bubble and the SN blast wave (e.g., Chevalier \& Liang 1989; Tenorio-Tagle et al. 1990, 1991; Dwarkadas 2005). Quantitative constraints on the density are difficult to obtain, however, since the radio luminosity cannot be related to the CSM density in the standard manner (i.e., self-similar solutions such that $L_{\nu} \propto \rho_{\mathrm{CSM}}^{0.4}-\rho_{\mathrm{CSM}}^{0.7}$ for $\alpha=0.79$; Chevalier 1996) and requires hydrodynamical simulations to account for the interaction properly.

Our constraints on $\tau_{\mathrm{ff}}$, on the other hand, are more straightforward to calculate. Using the Levenberg-Marquardt leastsquares method we directly constrain $\tau_{0}=\tau_{\mathrm{ff}}(\nu / 1 \mathrm{GHz})^{2.1}$ to be $29.3 \pm 6.1,15.8 \pm 1.1,4.1 \pm 0.6,0.69 \pm 0.10$, and $0.57 \pm 0.11$ for the 1996 December, 1997 December, 1999 June, 2004 April, and 2006 June epochs, respectively. Unfortunately, the 1996 August and 1997 June epochs only have $8.5 \mathrm{GHz}$ measurements and do not provide strong constraints, although we are encouraged by the fact that the latter is consistent with our adopted light-curve model. For the five epochs where we have good spectral constraints, we solve for $\rho_{\mathrm{CSM} \text {,shell }}$ explicitly in four contiguous shells such that

$$
\rho_{\mathrm{CSM}, \text { shell }} \approx 3.49 \times 10^{3}\left(\frac{T_{e}}{10^{4} \mathrm{~K}}\right)^{1.35}\left(\frac{\tau_{0}^{\text {in }}-\tau_{0}^{\text {out }}}{\int_{r^{\text {in }}}^{r_{\text {out }}} f(r) d r}\right)^{0.5} \mathrm{~cm}^{-3}
$$

where we have assumed $\mu_{\mathrm{H}}=0.6$ and $T_{e}=10,000 \mathrm{~K}$. The inner and outer radii $r^{\text {in }}$ and $r^{\text {out }}$, in $\mathrm{pc}$, are taken from $v_{s} t{ }^{24}$ we note that the outermost radius is constrained to be $\approx 2.8 \times 10^{17} \mathrm{~cm}$ on 2007-06-24 from our VLBI observation. The to-be-determined $r$-dependence of $\rho_{\mathrm{CSM}, \mathrm{r}}$ is encompassed

\footnotetext{
${ }^{24}$ While a self-similar solution is unlikely to hold under our current physical conditions, it is instructive to note that in such a scenario, the radii and velocity would evolve as $r=\left(v_{s} / m\right) t$ and $v_{s} \propto t^{-m}$, where $m$ will range between $0.85-0.95$ for most cases. This would lead to estimated shell radii only $\sim 20 \%$ larger and should highlight the relative quality of our radial constraints.
}

by $f(r)$. The form of $f(r)$ is iteratively determined by ensuring that it is consistent with the slope obtained between adjacent shells of $\rho_{\mathrm{CSM} \text {,shell }}$.

We next turn to the evolution of the shock velocity, $v_{s}$, which we need in order to determine the shell radii. From our optical spectrum, the ejecta (as traced by the $\mathrm{O}$ lines) and broad $\mathrm{H} \alpha$ line appear to be expanding at a variety of velocities between $\approx 2000-5800 \mathrm{~km} \mathrm{~s}^{-1}$, with the symmetric red and blueshifted velocities of $\approx 3250-3550 \mathrm{~km} \mathrm{~s}^{-1}$ standing out as the most likely values tracing the blast wave. We thus adopt $3400 \mathrm{~km} \mathrm{~s}^{-1}$ for the current shock speed. Clearly after the shock impacts a high-density region, its velocity will be reduced considerably. During its subsequent evolution in such a region, however, its velocity should evolve strongly, as can be seen from radio observations of SN 1987A (Manchester et al. 2002; Gaensler et al. 2007) and simulations thereof (Dwarkadas 2007b). Therefore, without any further information, a first approximation is to assume a constant velocity comparable to that derived from the optical spectrum. Interestingly, the high X-ray temperature, if it is in thermal equilibrium, also yields an equivalent velocity of $\approx 3400 \mathrm{~km} \mathrm{~s}^{-1}$, although here the velocity is almost certainly related to the reverse rather than forward shock and thus may simply be coincidence.

We therefore adopt an evolution for the blast wave velocity as follows. The shock began with a high initial speed which it maintained for 1-2 yrs. By 1996 August it encountered a dense shell of wind-swept material, and the shock velocity dropped rapidly by a factor proportional to the square root of the density gradient it encountered. This density gradient also initiated a rise in the radio luminosity by a factor of $>200$. Beyond 1996 August, we assume the velocity quickly arrived at a constant value of $\sim 3400 \mathrm{~km} \mathrm{~s}^{-1}$. These assumptions allow us to determine approximate radii of $0.054 \mathrm{pc}$, $0.055 \mathrm{pc}, 0.059 \mathrm{pc}, 0.064 \mathrm{pc}, 0.081 \mathrm{pc}$, and $0.089 \mathrm{pc}$ for the 1996 August, 1996 December, 1997 December, 1999 June, 2004 April, and 2006 June epochs, respectively.

If we take the radius on 1996 August as our shell impact date, then, depending on the 380 day ambiguity in the explosion date, the average initial shock velocity prior to this is constrained to be $\sim 37,000-139,000 \mathrm{~km} \mathrm{~s}^{-1}(0.12 c-0.46 c)$. The lower value is in line with theoretical and observational expectations (e.g., Chevalier et al. 2006), while the upper value is probably unrealistic. Such a high average shock speed would be difficult to maintain for more than several days in a typical or even underdense CSM environment. If the explosion did come later, then one or more of our assumptions above may be incorrect. The late-time velocity of SN 1996cr could be larger than our adopted value: the largest observed velocity in the optical spectrum is $\approx 5,800 \mathrm{~km} \mathrm{~s}^{-1}$, for instance, and provides a plausible upper limit to the current shock speed; adopting this value yields initial velocity constraints a factor of $\approx 3$ lower. Alternatively, the transition between initial and final velocities could have been gradual, taking many weeks to years to fully drop from one extreme to the other: the proposed CSM shell of SN 1996cr could have asymmetries akin to those seen in SN 1987A's ring, for instance, which are likely responsible for the onset of hotspots (e.g., Sugerman et al. 2002); a long-lived velocity transition could again lower initial velocity constraints by a factor of a few. Finally, the VLBI radius constraint could be too large, due either to our adopted ring model or our adopted distance to SN 1996cr: we note that uncertainties in our adopted VLBI model are esti- 


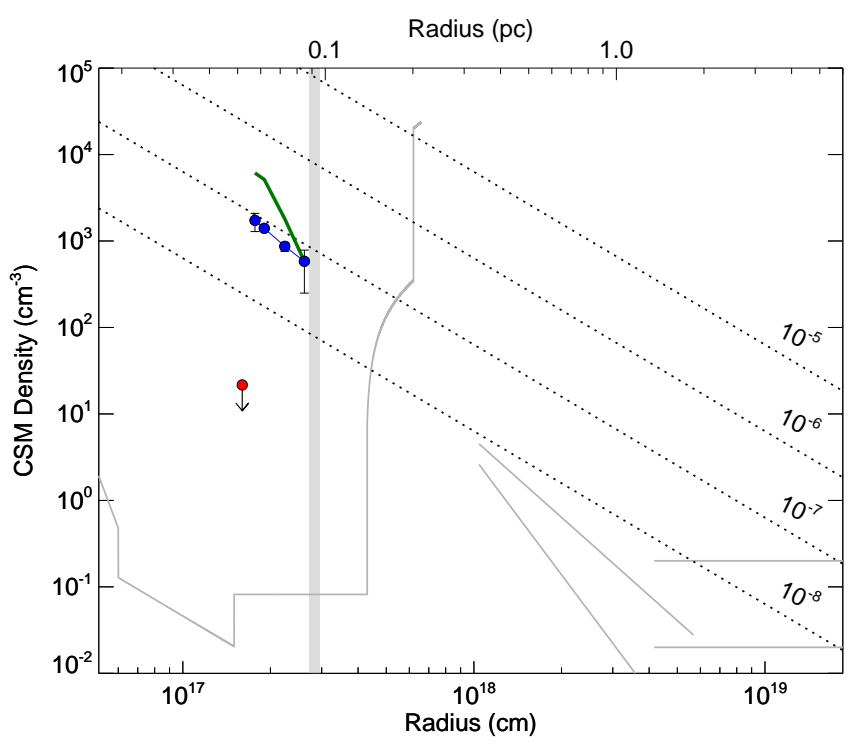

FIG. 12.— Estimated CSM density profile for SN $1996 \mathrm{cr}$; see $\S 4.3$ for full details. Radii are determined assuming a constant velocity of $3400 \mathrm{~km} \mathrm{~s}^{1}$ since 1996 December, anchored by our VLBI size constraint shown as a thick grey line. The CSM densities are estimated from the decreasing freefree absorption coefficient assuming a fully-ionized CSM (see 4.3 for details), and hence only trace the ionized portion of the CSM at these radii. The green curve denotes the density profile obtained assuming $\rho_{\mathrm{CSM}} \propto r^{-2}$, which is clearly inconsistent with this assumption. The blue points and curve show the density profile when modeled in a self-consistent manner, such that $\rho_{\mathrm{CSM}} \propto r^{-s}$, where $s$ is the iteratively determined slope between subsequent data points to be $-2.55,-2.93$, and -2.88 , respectively. We caution, however, that the exact slope $s$ depends intimately upon our adopted velocity evolution, estimated radio size, and adopted distance. The early upper limit (red) is the required density jump needed to reconcile the average estimated initial shock velocity with the much smaller average velocities inferred from the optical spectrum, assuming the density represented by the last measured point is fully ionized. The dotted lines denote constant values of $\dot{M} / v_{\mathrm{w}}$ in units of $\mathrm{M}_{\odot} \mathrm{yr}^{-1} \mathrm{~km}^{-1} \mathrm{~s}$ and bracket the typical values for red supergiant $\left(10^{-5}\right)$ and Wolf-Rayet $\left(10^{-8}\right)$ winds. For comparison, we also show the crude density profile (thin grey curves) for SN 1987A: the inner segment (left) is the density structure required to explain both the X-ray and radio emission (Dwarkadas $2007 \mathrm{c}$ ), while the outer lines (right) denote the upper and lower bounds on the density structure as deduced by light echoes (Sugerman et al. 2005).

mated to be only $\sim 5-20 \%$, while our adopted distance could vary by a factor of $\lesssim 2.7$ at most. Despite these potential problems, though, it is encouraging that our overall velocity model, assuming the lower initial shock velocity at least, is remarkably similar to the actual time evolution of the shock velocity in SN 1987A as deduced from radio observations (e.g., Manchester et al. 2002). In SN 1987A, the shock expanded at $\sim 35,000 \mathrm{~km} \mathrm{~s}^{-1}$ for its initial three years, before impacting with the $\mathrm{H}$ II region and slowing to $\sim 4500 \mathrm{~km} \mathrm{~s}^{-1}$.

Using Equation 6, we can now place constraints on $\rho_{\mathrm{CSM}, \mathrm{r}}$ in each shell of material overtaken between the five epochs as shown in Fig. 12. Since we do not know how far out the remainder of the free-free absorbing material extends into the CSM, we do not include the last $\tau_{0}$ value in this analysis. $H \alpha$ observations from the Southern H-Alpha Sky Survey Atlas (Gaustad et al. 2001) provide constraints on the emission measure toward the Circinus Galaxy of $\approx 10,000-25,000 \mathrm{pc} \mathrm{cm}^{-6}$, or equivalently $\tau_{0}=(0.0033-0.0082)\left(T_{e} / 10^{4} K\right)^{-1.35}$. Thus for any reasonable temperature of the warm interstellar medium (ISM), it appears that much of the remaining free-free absorption from our last measurement probably resides close to the $\mathrm{SN}$ in the CSM itself. Future low-frequency radio measurements could constrain this potential decline in the absorption.
Oddly, we find that the $\rho_{\mathrm{CSM}, \mathrm{r}} \propto r^{-s}$ profile derived from the absorption, where $s \approx 2.5-2.9$, seems at odds with the flat late-time evolution of the radio luminosity itself, which would naively imply $s \approx 1 .^{25}$ The strong disconnect between the two may indicate that a large portion of the CSM being overtaken is actually neutral, and thus not traced by the radio absorption. Since recombination is a strong function of density, this disconnect may further imply that there is significant clumping within the CSM of SN 1996cr.

Although we are unable to constrain the density profile encountered by the blast wave at early times via $\tau_{\text {ff }}$, the early implied velocity drop argues for a density increase of $\gtrsim 120$, while the radio luminosity jump supports a similar increase (even larger if $L_{\nu} \propto \rho_{\text {CSM }}^{0.4}-\rho_{\text {CSM }}^{0.7}$ holds). Thus it appears that we are seeing the transition between two distinctly different density regions separated by a dense shell, possibly associated with a wind-blown bubble (e.g., Dwarkadas 2005). If we assume the SN exploded into a sparse stellar wind $\left(\rho_{\mathrm{CSM}} \propto r^{-2}\right)$ initially, then we might expect the shock to adhere to a selfsimilar solution prior to 1996 August (i.e., radio emission evolves as $L_{\nu} \propto t^{-0.5}-t^{-1.5}$; CF94, Chevalier 1996). Thus for explosion dates earlier than 1995-06-07 (i.e., day 98 for $\left.t_{0}=1995-02-28\right)$, our $8.5 \mathrm{GHz}$ upper limit would constrain the CSM density in this region to be even lower still if no further SSA or FF absorption is present. A lower density, however, would imply a substantially larger density jump later on, which is difficult to reconcile with our current shock velocity evolution. This suggests that either the SN went off after this date, the early density did not follow a $r^{-2}$ profile, or there was additional heavy absorption that affected even the $8.5 \mathrm{GHz}$ data.

At the assumed radii, the CSM density of the shells equates to $\sim 0.05 M_{\odot}$ located between $(1.7-2.8) \times 10^{17} \mathrm{~cm}$. Additionally, the SN blast wave took only $\approx 1-2$ yr to reach this highdensity region, equating to $\sim(1-2) v_{\mathrm{s}} / v_{\mathrm{w}} \mathrm{yr}$ in the progenitor time frame. For reasonable wind velocities, this implies the region was formed a mere $\sim 70-14,000 \mathrm{yr}$ prior to the explosion.

For comparison, the density for a constant progenitor wind (i.e., constant $\dot{M} / \nu_{\mathrm{w}}$ ) can be written as

$$
\rho_{\mathrm{CSM}}=6.3 \times 10^{-12}\left(\frac{\dot{M}}{\mathrm{M}_{\odot} \mathrm{yr}^{-1}}\right)\left(\frac{v_{\mathrm{w}}}{\mathrm{km} \mathrm{s}^{-1}}\right)^{-1}\left(\frac{r}{10^{16} \mathrm{~cm}}\right)^{-2}
$$

and a few lines of constant $\dot{M} / v_{\mathrm{w}}$ are plotted in Fig. 12. Notably, the evolution of SN $1996 \mathrm{cr}$ beyond $\sim 10^{16} \mathrm{~cm}$ appears to be consistent with a constant wind of $\dot{M} / \nu_{\mathrm{w}} \sim 10^{-7}-10^{-8}$, typical of a Wolf-Rayet (WR) stage. However, it seems to have transitioned to a much sparser wind of $\dot{M} / v_{\mathrm{w}} \lesssim 10^{-9}$ before this (i.e., during its last many decades/centuries).

There are very few scenarios that can lead to such a CSM density distribution, with an initial low density, followed by a large density jump, and then a region of decreasing density. Such a distribution is reminiscent, however, of a wind-blown bubble, wherein the interaction of a star's stellar wind with either a previous stage of the stellar wind or with a constantdensity medium such as the ISM, results in material being swept up into a thin dense shell between the two regions. Go-

\footnotetext{
25 Radiation losses or strong gradients in the magnetic field could change $s$ substantially (e.g., Fransson \& Björnsson 1998). Note, however, than any additional loss mechanism would have to leave the late-time spectrum relatively unperturbed.
} 
ing outwards in radius, the bubble density distribution is described by (Weaver et al. 1977; Dwarkadas 2005): A region of freely expanding wind decreasing as $r^{-2}$, the wind termination shock, a constant-density region of shocked wind, the thin dense shell of swept-up ambient material and the outer medium, which could be another wind region with density decreasing as $r^{-2}$. The circumstellar distribution derived from the radio observations seems to fit this well, with the highdensity jump denoting the location of the dense shell, which we may not have seen from free-free absorption if the dense shell was not highly ionized. Interior to the shell is the low density shocked and unshocked wind, for which we only have upper limits. Exterior to the shell is the unshocked ambient medium, presumably also a wind, which we verify from our free-free absorption. If true, this picture again indicates that the stellar parameters changed significantly just a few years to a few thousand years before the death of the star, and that this region was formed by wind-wind interaction. Although the shell is thin, it is extremely dense, and the shock colliding with this region could be slowed dramatically, and lead to an increase in radio and X-ray emission, as is observed.

SN 1996cr can be compared to other observed young RSNe, among which SN 1987A (see McCray 2005, 2007, for reviews) is again a good starting point because of its rising radio light curve. SN 1987A was detected at radio wavelengths when it first went off, but this initial outburst was very shortlived (Turtle et al. 1987) and attributed to shock acceleration of synchrotron-emitting electrons in the stellar wind close to the star at the time of the explosion (Storey \& Manchester 1987; Chevalier \& Fransson 1987). Radio emission was again detected from the supernova after $\sim 3 \mathrm{yr}$ in quiescence, at first rising in a dramatic fashion for several hundred days and increasing more or less monotonically thereafter (see Fig. 6). Unlike the early absorption in SN 1996cr, however, the observed spectral index of SN 1987A has remained relatively constant between -1.0 to -0.9 , indicating unabsorbed synchrotron emission at all times during this re-emergence (Manchester et al. 2002). The steady rise in SN 1987A is also much steeper than in SN $1996 \mathrm{cr}$ and $\gtrsim 10^{4}$ times fainter, indicating a markedly different CSM-density distribution, and perhaps sharper density contrasts. The constant monitoring at radio, optical, and X-ray wavelengths has illuminated the density structure surrounding SN 1987A and directly confirmed multiple evolutionary stages of mass loss (Chevalier \& Dwarkadas 1995; Sugerman et al. 2005; Dwarkadas 2007a,b). Unfortunately such consistent monitoring is lacking for SN $1996 \mathrm{cr}$, leaving us with only a crude understanding of its density structure to date. Clearly the CSM distribution around SN 1996cr appears to be much denser and more compact than SN 1987A, in addition to potentially showing a pileup of material around $\sim 10^{17} \mathrm{~cm}$. We note that more modest structure in the CSM density, perhaps from the wind-wind interaction of multiple evolutionary stages or inhomogeneities within a particular stage, has been observed in many wellsampled RSNe light curves now, including SN 1978K (Smith et al. 2007a), SN 1979C (Montes et al. 2000), SN 1980K (Montes et al. 1998), and SN 1993J (Bartel et al. 2002; Weiler et al. 2007), and demonstrates that perturbations from $\rho_{\mathrm{CSM}} \propto$ $r^{-2}$ are probably commonplace. With finer sampling in the future, we should be able to place stronger constraints on the outlying density structure in SN 1996cr.

\subsection{X-ray Light Curve}

We turn next to the X-ray light curve of SN 1996cr. The Circinus Galaxy was observed with a variety of X-ray instruments as shown in Fig. 6 and Table 5. From these, we find that SN 1996cr exhibits strong, atypical temporal evolution. Unlike most SNe, SN 1996cr is not detected at early times despite relatively frequent and sensitive observations. Since thermal X-ray emission effectively traces the density of the CSM, these early X-ray upper limits, especially when compared to the detections later on, again indicate the presence of either strong early absorption $\left(N_{\mathrm{H}} \gtrsim 2 \times 10^{22} \mathrm{~cm}^{-2}\right)$ or a low-density cavity immediately surrounding the progenitor. Our radio constraints suggest in fact that both are present and likely to affect the X-ray emission: the mass associated with the FF absorption is already marginally enough to obscure an extrapolation of the detected X-ray slope, while the lower density implied by the radio luminosity jump would also result in decreased early X-ray emission. When finally detected on 2000-01-16, the 0.5-2 and 2-10 keV X-ray fluxes are bestfitted as $\propto t^{1.02 \pm 0.17}$ and $t^{0.80 \pm 0.10}$, respectively. The slopes are consistent with each other to within the errors, suggesting that any apparent emerging soft component is marginal and that the detected portion of the rise is likely to be optically thin. This strong, continued rise is in stark contrast to the evolution of nearly all other $\mathrm{SNe}$ detected to date, which are theoretically expected to decline as $\propto t^{-1}-t^{-0.4}$ (e.g., CF94) and observationally follow suit albeit with more scatter (e.g., see Fig. 2 of Immler \& Kuntz 2005). Sustained increases in the X-ray have only been observed for SN 1987A (Park et al. 2006), SN 2006jc (Immler et al. 2008), and perhaps marginally for SN 1978K (based upon a single early upper limit; Schlegel et al. 2004).

The X-ray luminosity of the forward and reverse shocks at a particular radius $r$ is given by

$$
L_{\mathrm{X}}=4 \pi \int \Lambda_{\mathrm{ff}}\left(T_{e, i}\right) n_{e, i}^{2} r_{i}^{2} d r
$$

where the index $i$ refers to values for either the forward or reverse shock and $\Lambda_{f f}$ is the temperature-dependent cooling rate (Chevalier \& Fransson 2003). The detection of strong He-like Fe and Si emission lines in the X-ray spectra (Bauer et al. 2001) suggests the presence of heavy elements in the $\mathrm{X}$-ray emitting material, and therefore that the X-ray emission stems from the shocked ejecta material found behind the reverse shock.

Because the progenitor CSM distribution likely includes the presence of pre-existing shocks and density discontinuities associated with the wind-blown bubble (see $\S 4.3$ ), the interaction of the SN blast wave with this medium is complex. Due to the presence of various discontinuities, the standard model (e.g Chevalier 1982b, ; CF94) of the interaction of a SN blast wave with a power-law ambient density medium is not applicable, and we must rely on detailed hydrodynamical models to infer the specific mass-loss properties of the progenitor (e.g., Dwarkadas 2005, 2007a,c). Furthermore the CSM may be at least partially photoionized, as is thought to be the case for SN 1987A (e.g., Chevalier \& Dwarkadas 1995). As shown in the previous section for a wind-blown bubble, even if the progenitor star had a constant wind mass-loss rate and velocity (which is not likely), the structure of the CSM can vary substantially with radius. Thus we cannot assume that the CSM density directly translates into the wind parameters. Nonetheless, we can make some qualitative arguments based on Equation 8. 
Between days 1800-4400, we find that the intrinsic X-ray luminosity scales almost linearly with time. The radius over this time period should increase by a factor of $\approx 1.6$, depending on the exact nature of the ejecta density profile and the CSM. The width of the emitting region can be approximated to be about 0.1 times the radius (e.g., CF94), resulting in an overall volume change by a factor of $\lesssim 5$. From the $\mathrm{X}$ ray spectrum, we do not see any significant changes in the temperature or absorption, indicating that $\Lambda_{f f}\left(T_{i}\right)$ remains approximately constant. If the X-ray emission was arising in the shocked ambient medium, the density in Equation 8 must decrease by a factor of $\approx 2.2$, or equivalently be $\propto r^{-1}$ to produce the observed dependence. This is significantly different from either a constant density or the $\approx r^{-2}$ dependence expected for a stellar wind with constant parameters. Furthermore, for a density profile decreasing as $r^{-1}$ the velocity would be expected to decrease with time, and therefore the temperature (which is proportional to $v^{2}$ ) would drop accordingly. The fact that this is not seen makes it unlikely that the X-ray emission is arising from the shocked wind (see Fig. 7).

We instead contend that the emission is arising from shocked ejecta behind (in a Lagrangian sense) the reverse shock. This scenario is strengthened by the presence of heavy element emission lines in the X-ray spectrum (cf. Fig. 6 of Bauer et al. 2001), since such elements are far more likely to reside in the processed ejecta than in the progenitor's stellar wind. If the $\mathrm{SN}$ ejecta did collide with a dense shell of CSM from a pre-existing wind-blown bubble, then the forward shock would advance quite slowly, and the bulk of the $\mathrm{X}$-ray emission would arise from the reverse-shocked material. The post-shock temperature just behind the reverse shock could be quite high $\left(\gtrsim 10^{9} \mathrm{~K}\right)$ if the density were low technically the density could drop to zero for expansion in a constant-density medium. The electron temperature, however, might be an order of magnitude lower if the electrons and ions have not yet reached equilibrium. The temperature would not change appreciably over this period, consistent with our observational constraints. The velocity of $\approx 3400 \mathrm{~km} \mathrm{~s}^{-1}$ inferred from the X-ray temperature in this case would refer to the electron temperature in the reverse shock, and thus the actual shock velocity, as traced by the ion temperature, could be considerably higher. Note that a similar linearly increasing light curve profile was found for the hard X-ray emission from SN 1987A (Park et al. 2005, 2006). Dwarkadas (2007a) has carried out numerical simulations that suggest that this emission arises from the reverse shocked ejecta, as had been postulated by Park et al. (2006). One puzzle which remains, though, is how the strong the $\mathrm{H} \alpha$ and Oxygen emission-line luminosities are powered. This discrepancy could be reconciled if there were holes in the CDS, such that a small fraction of the X-ray emission could escape while the rest went into exciting the strong optical line emission.

We can again gain some further insight by comparing SN 1996cr to other SNe. As stated above, SN 1987A has the most similarities. Like SN 1996cr, SN 1987A was undetected at X-ray wavelengths when it first went off, thereby excluding an explosion directly into a slow, dense wind that is characteristic of a red supergiant. Notably, SN 1987A was detected 130 days after explosion (Dotani et al. 1987; Sunyaev et al. 1987), although the X-ray emission here was attributed solely to radioactivity and appeared to rise and fall over the following year. After this brief period, the X-ray emission went undetected for another $\approx 3 \mathrm{yrs}$ before resurfacing above the noise
(Hasinger et al. 1996). Since this time, the X-ray emission has continued to rise as shown in Fig. 6, with its light curve well-modeled by a broken power law (Park et al. 2006). The reappearance of the emission at about 3 years was postulated as due to the interaction of the $\mathrm{SN}$ blast wave with a region of ionized wind material from the progenitor (Chevalier \& Dwarkadas 1995). They suggested that this region is material photoionized from the dense circumstellar shell that surrounds SN 1987A, formed as a result of the interaction of the progenitor blue supergiant wind with a pre-existing red supergiant wind. Although the evolution of the star may be different, a physical scenario broadly like that of SN 1987A, with a region formed by mass-loss from a progenitor star, certainly seems plausible for SN 1996cr, although the contrasting lightcurve slopes, luminosities, and radial distances clearly highlight the different CSM density structure. The only other SN to exhibit a rise at X-ray wavelengths is SN 2006aj, a peculiar type Ib. The light curve of SN 2006aj has been ascribed to the interaction of the blast wave with a dense shell of material left over from an LBV-like outburst of the SN progenitor $~ 2$ years prior to the explosion (Immler et al. 2008).

The X-ray spectrum for SN 1996cr, moreover, is modeled by a hot thermal plasma $(k T=13.4 \mathrm{keV})$ similar to the early phase of SN 1993J (Zimmermann \& Aschenbach 2003) but substantially hotter than SN 1987A $(k T \approx 3 \mathrm{keV}$ with an emerging $0.3 \mathrm{keV}$ component), and implies that the conditions within the X-ray-emitting region are somewhat different. The hotter temperature could indicate a higher shock velocity, or a larger level of equilibration between the electrons and ions. The X-ray spectrum of SN 1996cr is additionally absorbed below $\sim 2 \mathrm{keV}$. While some fraction of the substantial non-Galactic column density could be due to self-absorption, we consider this to be minimal given that other X-ray point sources in the Circinus Galaxy have similar X-ray derived column densities (Bauer et al. 2001).

The rapid evolution we see here for SN 1996cr has been noted in a few other objects, such as SN 2002kg (Van Dyk et al. 2006; Maund et al. 2006) and SN 2006jc (Foley et al. 2007; Pastorello et al. 2007). Such an evolution has also been invoked to explain the enormous energy output from SN 2006gy (Ofek et al. 2007; Smith et al. 2007b), although the lack of obvious CSM-interaction measures at X-ray, $\mathrm{H} \alpha$, and radio wavelengths here implies that perhaps something more extreme is going on (e.g., Smith \& McCray 2007c). Unfortunately, for many of the older, classic X-ray emitting type IIn SNe such as SN 1978K (Schlegel et al. 2004; Smith et al. 2007a), SN 1979C (Immler \& Kuntz 2005), and SN 1986J (Temple et al. 2005), early-time behavior of the Xray emission remains completely unknown and thus the frequency and duration of sudden evolutionary changes in $\mathrm{SNe}$ progenitors is still an open question. The X-ray evolution of SN 1996cr may allow us to explain the relatively flat late-time X-ray light curves for objects like SN 1978K and SN 1979C, for instance.

\section{CONCLUSIONS}

We have confirmed SN 1996cr as one of the nearest, and subsequently X-ray and radio-brightest, $\mathrm{SNe}$ to date. Archival optical imaging data have constrained the explosion date to within $\approx 1$ yr prior to 1996-03-16, while a new VLT spectrum has identified it as a type IIn SN. The multi-wavelength constraints detailed above point to a rich progenitor history. As is the case for most type IIn SNe, SN 1996cr appears to have originated from a massive star that shed its outer layers 
at some late evolutionary stage, which now act as the target for the outgoing SN shock.

The optical light curve hints at a mild re-brightening at late times, possibly driven by line emission, or by X-ray photons being downscattered and being emitted as optical photons. From the recent optical spectrum, the strong narrow $\mathrm{H}$ emission points to a significant accumulation of mass in the CSM prior to the SN. We are additionally able to resolve the complex $\mathrm{O}$ emission into a number of distinct high-velocity components with FWHM $\sim 2000-3000 \mathrm{~km} \mathrm{~s}^{-1}$. The origin of such distinct $\mathrm{O}$ complexes remains somewhat unclear, perhaps arising from one or more of the following: structural asymmetries, differential absorption, or multiple concentric shocks.

The X-ray and radio emission allow us to quantify the CSM density, tracing out two apparently distinct regions, which cannot be reconciled with a single constant-wind model. From the radio and X-ray upper limits, we infer that the inner region $\left(r \lesssim 1 \times 10^{17} \mathrm{~cm}\right)$ is likely quite sparse. The outer region $\left[r \approx(1.5-2.8) \times 10^{17} \mathrm{~cm}\right]$, as traced by the early radio absorption, radio luminosity jump, and X-ray emission, is not well constrained, and is likely associated with the interaction region between two distinct progenitor winds, which culminates in a thin, dense wind-swept shell. This second region appears relatively dense $\left(\rho_{\mathrm{CSM}} \approx 10^{3} \mathrm{~cm}^{-3}\right)$, potentially consistent with a slow-velocity WR-like wind, although the CSM density profile traced by the radio absorption $\left(\rho_{\mathrm{CSM}} \propto r^{-2.5}\right)$ naively seems at odds with the implied CSM density profiles from the radio and X-ray luminosities $\left(\rho_{\mathrm{CSM}} \propto\right.$ constant or $r^{-1}$, respectively). A more detailed understanding of the CSM awaits sophisticated hydrodynamical models.

The most plausible explanation is one where the progenitor changed evolutionary states just prior to explosion, likely transitioning from a WR-like wind to an even faster, less dense wind, resulting in portions of the previous CSM being swept-up to form a wind-blown cavity surrounded by a dense shell. Such a scenario has been recently proposed for the peculiar type Ib SN 2006aj (Foley et al. 2007; Pastorello et al. 2007; Immler et al. 2008). The radio and X-ray emission are constant or still rising, demonstrating that the blast wave is still making its way through the dense, swept-up shell at the edge of the cavity. The collision of the blast wave with the shell would result in a transmitted shock expanding into the shell, and a reflected shock expanding back into the SN ejecta. This reflected shock should be distinguished initially from the reverse shock of the SN expanding into the ejecta, although it will eventually overtake the reverse shock. The overall effect could be one of several shocks and rarefaction waves travelling back and forth through the ejecta. A spectrum would reveal several different velocity structures, perhaps as we observe in the $\mathrm{O}$ lines. The reason why it is seen mainly in $\mathrm{O}$ is unclear, but it could indicate that this is an $\mathrm{O}$ rich remnant, similar to Cas A for example (see Fesen et al. 2001, and references therein). Or perhaps it is just that the reverse shock has not penetrated very far into the ejecta, so that only the O-rich layers have been shocked. We note that if the shock is indeed progressing into shell of constant density, then the resulting density gradients would not lead to the formation of a CDS at all (e.g., see Fig. 1-4 of Chevalier 1982a). If so, then the observed $\mathrm{O}$ velocity components may instead arise from a disk or ring prehaps similar to SN 1987A.

Once the blast wave makes its way through the dense shell, it will emerge from the other side, presumably as a weak shock. This should encounter a relatively pristine wind and start to decline in both the radio and X-ray bands. Further regular monitoring of SN $1996 \mathrm{cr}$ should reveal this. Once this happens, we will be able to strengthen many of our current constraints and assumptions.

We have compared SN 1996cr to the late-time behavior of SN 1987A, which is the only other SN with a documented dramatic increase at X-ray and radio wavelengths. SN 1996cr is likely propagating through its CSM in a manner similar to SN 1987A expanding into its H II region and circumstellar ring (e.g., Park et al. 2006). The X-ray and radio luminosities of SN 1996cr, however, are several orders of magnitude brighter and comparable to typical type IIn SNe such as SN $1978 \mathrm{~K}$ and SN 1979C. This is presumably because the swept-up CSM is more compact, and perhaps, unlike SN 1987A, there is no intervening high-density material encountered which slows down the ejecta until they reach a dense shell, so the density jump is higher and subsequently more dramatic. As we follow SN 1996cr over the next few decades, we anticipate that it will transition into an evolution similar to other SNe. It is intriguing to think that SN $1996 \mathrm{cr}$ might represent a sort of "bridge" object between the extremes of SN 1987A and these more luminous type II SNe. Although plagued by small number statistics, the fact that two (although perhaps more if we count SN 1978K and SN 1979C) of the $\sim 5-10$ closest $\mathrm{SNe}$ to have exploded in the past four decades show evidence for wind-blown bubbles implies that the phenomenon is common, and may indicate that current $\mathrm{SNe}$ searches are somehow biased against them at larger distances. Systematic constraints exist only for type Ibc SNe (Soderberg et al. 2006a,b), where late-time radio observations indicate that only $\sim 2 \%$ of initially undetected targets show late-time detections, while $\sim 40 \%$ of detections exhibit abrupt light-curve variations.

The large mass associated with the pre-SN CSM surrounding SN 1996cr could have been the result of explosive mass loss thousands of years before the SN explosion (e.g., Woosley et al. 2002; Heger \& Woosley 2002), qualitatively similar to SN 2006aj (Foley et al. 2007; Pastorello et al. 2007; Immler et al. 2008), although the limited X-ray and radio data point to a relatively smooth density profile, which implies a more gradual process was at work. Numerous other SNe including SN 1994W (Chugai et al. 2004), SN 2001em (Chugai \& Chevalier 2006; Bietenholz \& Bartel 2007), SN 2006gy (Ofek et al. 2007; Smith et al. 2007b), and SN 2006jc (Foley et al. 2007; Pastorello et al. 2007; Immler et al. 2008) all show signs of dense, enriched CSM likely produced by giant mass-loss events just prior to their SNe, implying that this form of mass-loss is relatively common. LBVs have often been invoked to account for such episodes of extreme massloss (e.g., Gal-Yam et al. 2007; Smith et al. 2007b). Indeed a giant outburst was seen in the case of SN 2006jc just 2 years prior to core-collapse (Pastorello et al. 2007). Again it is unclear whether the explosive nature of LBVs can be reconciled with the relatively smooth mass-loss/density gradients observed for SN 1996cr.

Our analyses clearly demonstrate that $\mathrm{SN} 1996 \mathrm{cr}$ is a unique and compelling target. Its proximity and flux enable a wealth of observations that would be otherwise impractical for typical SNe. We have reported on initial findings of a VLBI campaign underway to resolve spatially SN 1996cr at several radio wavelengths, eventually enabling studies of its morphology and temporal expansion. This will provide a more coherent picture, allowing us to set strong limits on the expansion velocity from the resolved size and potentially con- 
firm the implied asymmetries we infer from the optical spectrum. Follow-up HST imaging will provide constraints on any potential light echoes in the vicinity of SN 1996cr, possibly allowing refined estimates of its explosion date, distance, and outlying CSM structure. Likewise, follow-up X-ray observations will hopefully elucidate the nature of the strong continued rise in the X-ray band and better constrain properties of the reverse shock region. Notably, SN 1996cr is bright enough to be efficiently monitored using the Chandra HETGS, enabling high-resolution studies of its numerous X-ray emission lines and their potential evolution; an in-depth discussion of the current X-ray spectral constraints, including a composite Chandra HETGS spectrum from the data presented here, are forthcoming (F. Bauer et al., in preparation). Additionally, future optical spectroscopic studies should allow investigations into the temporal behavior of complex emission lines found in our discovery spectrum. Infrared imaging and spectroscopy will help address the potential for dust formation; notably type II SNe are thought to contribute strongly to the overall dust content of galaxies. Looking ahead to the near future, if SN 1996cr remains relatively bright at millimeter wavelengths, we anticipate that observations with ALMA will provide a substantial leap in terms of understanding the structure and composition of SN 1996cr and its relation to both SN 1987A and more typical type II SNe.

If any researchers have further serendipitous multiwavelength observations of SN 1996cr during the critical 1995-1996 period, we would be grateful to learn of these.
We thank Bjorn Emonts and Bryan Gaensler for help in reducing archival ATCA observations, Roger Chevalier, Claes Frannson, Dick McCray, Kurt Weiler, and Christopher Stockdale for useful discussions about the nature of the multiwavelength emission, Baerbel Koribalski for increasing our awareness of Australian online archives and access to proprietary ATCA observations, Ernesto Oliva and Alessandro Marconi for access to reduced NTT data, Bruno Leibundgut and Rob Fesen for their spectra of SN 1986J and SN 1979C, Philip Edwards, Steven Tingay, and Tasso Tzioumis for support of the VLBI observations, Kevin Hurley for directing us to revised IPN results which allowed us to reject an otherwise tentative GRB identification, John Raymond for helping to improve our emission-line tables, and the anonymous referee for useful comments that improved the content and presentation of the paper. We gratefully acknowledge the financial support of Chandra Postdoctoral Fellowship Award PF4-50032 (FEB), NSF award AST-0319261 (VVD), NASA STScI grant HST-AR-10649 (VVD), NASA LTSA grant NAG5-13035 (WNB), the Leverhulme Trust (SJS) and the ESF EURYI scheme (SJS).

Facilities: ATCA, ASCA (GIS, SIS), AAT (TAURUS), BeppoSAX (LECS, MECS), CGRO (BATSE), CXO (ACIS, HETGS), Hobart, HST (WFPC2, NICMOS), Max Planck:2.2m (IRAC2) Mopra, NTT (SUSI), Parkes ROSAT (HRI), Swift (UVOT, XRT), UKST, VLT (FORS), XMM (pn, MOS),

\section{REFERENCES}

Aldering, G., et al. 2006, ApJ, 650, 510

Aretxaga, I., Benetti, S., Terlevich, R. J., Fabian, A. C., Cappellaro, E., Turatto, M., \& della Valle, M. 1999, MNRAS, 309, 343

Arnaud, K. A. 1996, in ASP Conf. Ser. 101: Astronomical Data Analysis Software and Systems V, vol. 5, 17

Bartel, N., et al. 2002, ApJ, 581, 404

Bauer, F. 2007, Central Bureau Electronic Telegrams, 879, 1

Bauer, F. E., Brandt, W. N., Sambruna, R. M., Chartas, G., Garmire, G. P., Kaspi, S., \& Netzer, H. 2001, AJ, 122, 182

Bertin, E. \& Arnouts, S. 1996, A\&AS, 117, 393

Bietenholz, M. F. \& Bartel, N. 2007, ApJ, 665, L47

Bietenholz, M. F., Bartel, N., \& Rupen, M. P. 2001, ApJ, 557, 770

Bland-Hawthorn, J. \& Jones, D. H. 1998, Publications of the Astronomical Society of Australia, 15, 44

Broos, P., Townsley, L., Getman, K., \& Bauer, F. 2005, ACIS Extract, An ACIS Point Source Extraction Package, Pennsylvania State University, http://www.astro.psu.edu/xray/docs/TARA/ae users guide.html

Burstein, D. \& Heiles, C. 1978, ApJ, 225, 40

Calzetti, D., Kinney, A. L., \& Storchi-Bergmann, T. 1994, ApJ, 429, 582

Cardelli, J. A., Clayton, G. C., \& Mathis, J. S. 1989, ApJ, 345, 245

Cash, W. 1979, ApJ, 228, 939

Chevalier, R. A. 1982a, ApJ, 258, 790

- 1982b, ApJ, 259, 302

- 1996, in Radio Emission from the Stars and the Sun, eds. A. R. Taylor \&

J. M. Paredes, vol. 93 of Astronomical Society of the Pacific Conference

Series, 125

- 2005, ApJ, 619, 839

Chevalier, R. A., Blondin, J. M., \& Emmering, R. T. 1992, ApJ, 392, 118

Chevalier, R. A. \& Dwarkadas, V. V. 1995, ApJ, 452, L45

Chevalier, R. A. \& Fransson, C. 1987, Nature, 328, 44

- 1994, ApJ, 420, 268

- 2003, in Supernovae and Gamma-Ray Bursters, ed. K. Weiler, vol. 598 of Lecture Notes in Physics, Berlin Springer Verlag, 171

Chevalier, R. A., Fransson, C., \& Nymark, T. K. 2006, ApJ, 641, 1029

Chevalier, R. A. \& Liang, E. P. 1989, ApJ, 344, 332

Chugai, N. N. 1991, MNRAS, 250, 513

Chugai, N. N. \& Chevalier, R. A. 2006, ApJ, 641, 1051

Chugai, N. N., et al. 2004, MNRAS, 352, 1213

Crotts, A. P. S. \& Heathcote, S. R. 2000, ApJ, 528, 426

Davis, J. E. 2001, ApJ, 562, 575
Deng, J., et al. 2004, ApJ, 605, L37

Dickey, J. M. \& Lockman, F. J. 1990, ARA\&A, 28, 215

Dotani, T., Hayashida, K., Inoue, H., Itoh, M., \& Koyama, K. 1987, Nature, 330,230

Dwarkadas, V. V. 2000, ApJ, 541, 418

- 2005, ApJ, 630, 892

- 2007a, in American Institute of Physics Conference Series, eds.

S. Immler \& R. McCray, vol. 937 of American Institute of Physics Conference Series, 120

- 2007b, in Revista Mexicana de Astronomia y Astrofisica Conference

Series, vol. 30 of Revista Mexicana de Astronomia y Astrofisica

Conference Series, 49

- 2007c, ApJ, 667, 226

Dwek, E., et al. 2008, ApJ, 676, 1029

Elmouttie, M., Koribalski, B., Gordon, S., Taylor, K., Houghton, S.,

Lavezzi, T., Haynes, R., \& Jones, K. 1998, MNRAS, 297, 49

Fassia, A., et al. 2001, MNRAS, 325, 907

Fesen, R. A., Morse, J. A., Chevalier, R. A., Borkowski, K. J., Gerardy, C. L., Lawrence, S. S., \& van den Bergh, S. 2001, AJ, 122, 2644

Fesen, R. A., et al. 1999, AJ, 117, 725

Filippenko, A. V. 1997, ARA\&A, 35, 309

Foley, R. J., Smith, N., Ganeshalingam, M., Li, W., Chornock, R., \& Filippenko, A. V. 2007, ApJ, 657, L105

Fransson, C., Benvenuti, P., Wamsteker, W., Gordon, C., Hempe, K.,

Reimers, D., Palumbo, G. G. C., \& Panagia, N. 1984, A\&A, 132, 1

Fransson, C. \& Björnsson, C.-I. 1998, ApJ, 509, 861

Fransson, C., et al. 2002, ApJ, 572, 350

- 2005, ApJ, 622, 991

Freeman, K. C., Karlsson, B., Lynga, G., Burrell, J. F., van Woerden, H., Goss, W. M., \& Mebold, U. 1977, A\&A, 55, 445

Gaensler, B. M., Staveley-Smith, L., Manchester, R. N., Kesteven, M. J., Ball, L., \& Tzioumis, A. K. 2007, in American Institute of Physics Conference Series, eds. S. Immler \& R. McCray, vol. 937 of American Institute of Physics Conference Series, 86

Gal-Yam, A., et al. 2007, ApJ, 656, 372

Garcia-Segura, G., Langer, N., \& Mac Low, M.-M. 1996a, A\&A, 316, 133 Garcia-Segura, G., Mac Low, M.-M., \& Langer, N. 1996b, A\&A, 305, 229

Garnavich, P. M., et al. 2003, ApJ, 582, 924

Gaustad, J. E., McCullough, P. R., Rosing, W., \& Van Buren, D. 2001,

PASP, 113, 1326 
Gerardy, C. L., Fesen, R. A., Höflich, P., \& Wheeler, J. C. 2000, AJ, 119, 2968

Gerardy, C. L., et al. 2002, ApJ, 575, 1007

Germany, L. M., Reiss, D. J., Sadler, E. M., Schmidt, B. P., \& Stubbs, C. W. 2000, ApJ, 533, 320

Gordon, S., Koribalski, B., Houghton, S., \& Jones, K. 2000, MNRAS, 315, 248

Hamuy, M., et al. 2003, Nature, 424, 651

Hasinger, G., Aschenbach, B., \& Truemper, J. 1996, A\&A, 312, L9

Heger, A. \& Woosley, S. E. 2002, ApJ, 567, 532

Hurley, K., et al. 1999, ApJS, 122, 497

Hurley, K., et al. 2005, ApJS, 156, 217

Immler, S. \& Kuntz, K. D. 2005, ApJ, 632, L99

Immler, S., et al. 2008, ApJ, 674, L85

Keenan, F. P., Crawford, F. L., Feibelman, W. A., \& Aller, L. H. 2001, ApJS, 132,103

Keenan, F. P., Kingston, A. E., \& Johnson, C. T. 1988, A\&A, 202, 253

Keenan, F. P. \& Norrington, P. H. 1987, A\&A, 181, 370

- 1991, ApJ, 368, 486

Kriss, G. 1994, in Astronomical Data Analysis Software and Systems III, eds. D. R. Crabtree, R. J. Hanisch, \& J. Barnes, vol. 61 of Astronomical Society of the Pacific Conference Series, 437

Lamers, H. J. G. L. M., Maeder, A., Schmutz, W., \& Cassinelli, J. P. 1991, ApJ, 368, 538

Langer, N., Hamann, W.-R., Lennon, M., Najarro, F., Pauldrach, A. W. A., \& Puls, J. 1994, A\&A, 290, 819

Leibundgut, B., Kirshner, R. P., Pinto, P. A., Rupen, M. P., Smith, R. C., Gunn, J. E., \& Schneider, D. P. 1991, ApJ, 372, 531

Leonard, D. C., Filippenko, A. V., Barth, A. J., \& Matheson, T. 2000, ApJ, 536, 239

Maeder, A. \& Meynet, G. 2000, ARA\&A, 38, 143

Manchester, R. N., Gaensler, B. M., Wheaton, V. C., Staveley-Smith, L., Tzioumis, A. K., Bizunok, N. S., Kesteven, M. J., \& Reynolds, J. E. 2002, Publications of the Astronomical Society of Australia, 19, 207

Marconi, A., Moorwood, A. F. M., Origlia, L., \& Oliva, E. 1994, The Messenger, 78, 20

Matheson, T., Filippenko, A. V., Ho, L. C., Barth, A. J., \& Leonard, D. C. 2000, AJ, 120, 1499

Matt, G., et al. 1999, A\&A, 341, L39

Maund, J. R., et al. 2006, MNRAS, 369, 390

McCray, R. 1993, ARA\&A, 31, 175

- 2005, in IAU Colloq. 192: Cosmic Explosions, On the 10th Anniversary of SN1993J, eds. J.-M. Marcaide \& K. W. Weiler, 77

- 2007, in American Institute of Physics Conference Series, eds. S. Immler \& R. McCray, vol. 937 of American Institute of Physics Conference Series, 3

Meegan, C. A., et al. 1996, ApJS, 106, 65

Montes, M. J., van Dyk, S. D., Weiler, K. W., Sramek, R. A., \& Panagia, N. 1998, ApJ, 506, 874

Montes, M. J., Weiler, K. W., Van Dyk, S. D., Panagia, N., Lacey, C. K., Sramek, R. A., \& Park, R. 2000, ApJ, 532, 1124

Nymark, T. K., Fransson, C., \& Kozma, C. 2006, A\&A, 449, 171

Ofek, E. O., et al. 2007, ApJ, 659, L13

Osterbrock, D. E. 1989, Astrophysics of gaseous nebulae and active galactic nuclei (Mill Valley: University Science Books)

Park, S., Zhekov, S. A., Burrows, D. N., Garmire, G. P., Racusin, J. L., \& McCray, R. 2006, ApJ, 646, 1001

Park, S., Zhekov, S. A., Burrows, D. N., \& McCray, R. 2005, ApJ, 634, L73

Pastorello, A., et al. 2002, MNRAS, 333, 27

- 2007, Nature, 447, 829

Patat, F. 2005, MNRAS, 357, 1161

Patat, F., Barbon, R., Cappellaro, E., \& Turatto, M. 1994, A\&A, 282, 731
Patat, F., Benetti, S., Cappellaro, E., \& Turatto, M. 2006, MNRAS, 369, 1949

Porter, R. L., Bauman, R. P., Ferland, G. J., \& MacAdam, K. B. 2005, ApJ, 622, L73

Pozzo, M., Meikle, W. P. S., Fassia, A., Geballe, T., Lundqvist, P., Chugai, N. N., \& Sollerman, J. 2004, MNRAS, 352, 457

Prieto, J. L., et al. 2008, AJ, submitted (astro-ph:0706.4088)

Rupen, M. P., van Gorkom, J. H., Knapp, G. R., Gunn, J. E., \& Schneider, D. P. 1987, AJ, 94, 61

Ryder, S., Staveley-Smith, L., Dopita, M., Petre, R., Colbert, E., Malin, D., \& Schlegel, E. 1993, ApJ, 416, 167

Schlegel, D. J., Finkbeiner, D. P., \& Davis, M. 1998, ApJ, 500, 525

Schlegel, E. M. 1990, MNRAS, 244, 269

Schlegel, E. M., Kong, A., Kaaret, P., DiStefano, R., \& Murray, S. 2004, ApJ, 603, 644

Smith, I. A., Ryder, S. D., Boettcher, M., Tingay, S. J., Stacy, A., Pakull, M. \& Liang, E. P. 2007a, ApJ, 669, 1130

Smith, N., et al. 2007b, ApJ, 666, 1116

Smith, N., \& McCray, R. 2007, ApJ, 671, L17

Smith, R. K., Brickhouse, N. S., Liedahl, D. A., \& Raymond, J. C. 2001, ApJ, 556, L91

Soderberg, A. M., et al. 2008, Nature, in press (astro-ph/0802.1712)

Soderberg, A. M., Chevalier, R. A., Kulkarni, S. R., \& Frail, D. A. 2006a, ApJ, 651, 1005

Soderberg, A. M., Nakar, E., Berger, E., \& Kulkarni, S. R. 2006b, ApJ, 638, 930

Storey, M. C. \& Manchester, R. N. 1987, Nature, 329, 421

Stothers, R. B. \& Chin, C.-W. 1996, ApJ, 468, 842

Sugerman, B. E. K. 2003, AJ, 126, 1939

Sugerman, B. E. K., Crotts, A. P. S., Kunkel, W. E., Heathcote, S. R., \& Lawrence, S. S. 2005, ApJS, 159, 60

Sugerman, B. E. K., Lawrence, S. S., Crotts, A. P. S., Bouchet, P., \&

Heathcote, S. R. 2002, ApJ, 572, 209

Sunyaev, R., et al. 1987, Nature, 330, 230

Temple, R. F., Raychaudhury, S., \& Stevens, I. R. 2005, MNRAS, 362, 581

Tenorio-Tagle, G., Bodenheimer, P., Franco, J., \& Rozyczka, M. 1990, MNRAS, 244, 563

Tenorio-Tagle, G., Rozyczka, M., Franco, J., \& Bodenheimer, P. 1991, MNRAS, 251, 318

Turatto, M., Benetti, S., \& Cappellaro, E. 2003, in From Twilight to Highlight: The Physics of Supernovae, eds. W. Hillebrandt \& B. Leibundgut, 200

Turatto, M., Cappellaro, E., Barbon, R., della Valle, M., Ortolani, S., \& Rosino, L. 1990, AJ, 100, 771

Turtle, A. J., Campbell-Wilson, D., Bunton, J. D., Jauncey, D. L., \& Kesteven, M. J. 1987, Nature, 327, 38

Van Dyk, S. D., Li, W., Filippenko, A. V., Humphreys, R. M., Chornock, R., Foley, R., \& Challis, P. M. 2006, PASP, submitted (astro-ph/0603025)

Veilleux, S. \& Bland-Hawthorn, J. 1997, ApJ, 479, L105

Wang, L., Baade, D., Höflich, P., Wheeler, J. C., Kawabata, K., \& Nomoto, K. 2004, ApJ, 604, L53

Wang, L., Wheeler, J. C., Li, Z., \& Clocchiatti, A. 1996, ApJ, 467, 435

Weaver, R., McCray, R., Castor, J., Shapiro, P., \& Moore, R. 1977, ApJ, 218,377

Weiler, K. W., Williams, C. L., Panagia, N., Stockdale, C. J., Kelley, M. T., Sramek, R. A., Van Dyk, S. D., \& Marcaide, J. M. 2007, ApJ, 671, 1959

Woosley, S. E., Heger, A., \& Weaver, T. A. 2002, Reviews of Modern Physics, 74, 1015

Zimmermann, H.-U., \& Aschenbach, B. 2003, A\&A, 406, 969 